\title{
ITERATIVELY REGULARIZED LANDWEBER ITERATION METHOD: CONVERGENCE ANALYSIS VIA HÖLDER STABILITY
}

\author{
Gaurav Mittal and Ankik Kumar Giri \\ Department of Mathematics, Indian Institute of Technology Roorkee, Roorkee, India, 247667. \\ gmittal@ma.iitr.ac.in, ankik.giri@ma.iitr.ac.in
}

\begin{abstract}
In this paper, the local convergence of Iteratively regularized Landweber iteration method is investigated for solving non-linear inverse problems in Banach spaces. Our analysis mainly relies on the assumption that the inverse mapping satisfies the Hölder stability estimate locally. We consider both noisy as well as non-noisy data in our analysis. Under the a-priori choice of stopping index for noisy data, we show that the iterates remain in a certain ball around exact solution and obtain the convergence rates. The convergence of the Iteratively regularized Landweber iterates to the exact solution is shown under certain assumptions in the case of non-noisy data and as a by-product, under different conditions, two different convergence rates are obtained.
\end{abstract}

AMS Subject Classifications: 65J15, 47A52, 47J06

Keywords: Iterative Regularization, Nonlinear ill-posed problems, Hölder stability estimates

\section{INTRODUCTION}

Let $F: D(F) \subset U \rightarrow V: F(u)=v$ be a non-linear forward operator between the Banach spaces $U$ and $V$. The classical meaning of an inverse problem is the determination of $u \in U$, provided $v$ or some approximation of $v$ is given. For further details on inverse problems, see [16] for Hilbert spaces settings, and [33] for Banach space settings. In general, due to the lack of continuous dependence on the data, almost all the inverse problems are ill-posed in nature. Thus, regularization methods are needed to find the stable approximate solutions of the ill-posed inverse problems. Variational regularization methods are well known regularization methods for finding the stable approximate solutions and are well studied, see, for instance, $[16,26]$. Nevertheless, iterative methods are often an appealing alternative to variational methods (specifically for large-scale problems). Among all the iterative methods, Landweber iteration method is one of the well known classical methods. For the convergence results of Landweber iteration and its modifications in Hilbert spaces, an extensive research has been done in [21,23]. In the case of monotone operators, there is an important role of duality mappings in iterative methods (see $[5,8,9,36]$ ). Using the duality mapping, non-linear generalization of the Landweber method is given in [10] for Banach spaces. Scherzer, in [25], gave the modification of Landweber iteration method and coined it as iteratively regularized Landweber iteration method. This method is highly motivated from the iteratively regularized Gauss-Newton method introduced 
by Bakushinskii in [1]. In our study, the data space $V$ can be any arbitrary Banach space but the model space $U$ needs to be uniformly convex and smooth (see next section for their formal definitions). In the theory of Banach spaces, Bregman distances play an important role because of their rich geometrical properties and are more convenient to employ rather than Ljapunov functionals to prove the convergence of regularization schemes [31]. And hence, it is more appropriate to derive the convergence rates with the help of Bregman distances.

Conceptually, convergence rates can be derived with two different approaches for non-linear problems. First one is on the basis of source and non-linearity conditions, see, for instance, $[16,26,27,33]$ for variational regularization, and $[2,3,4,30,33]$ for iterative regularization. The second approach relies on the stability estimates which has been derived in [18] for Tikhonov's regularization method and in [24] for iterative regularization (Landweber iteration method) in Banach spaces. The results regarding the rates of convergence using Hölder stability estimates and logarithmic stability estimates can also be found in $[12,15]$ and $[34,35]$ respectively.

In our analysis, we consider the iteratively regularized Landweber iteration scheme which is taken from [33]. The motivation for this paper comes from [24] in which the convergence rates for Landweber iteration method have been obtained via Hölder stability estimates, however, only non-noisy data is considered there. The prime motive of this work is to study the convergence of the iterates of Iteratively regularized Landweber iteration method (2.1)-(2.2) provided the inverse mapping satisfies the Hölder stability estimate (3.2) and hence find the convergence rates. Since non-noisy data is taken in [24] for Landweber iteration method, we want to emphasize that from our results, one can also deduce the convergence rates in the presence of noisy data for Landweber iteration method. Novelty of this work is to determine the convergence rates for both the noisy as well as non-noisy data without using the classical approach based on source conditions as well as the contemporary smoothness concept known as variational inequalities.

The plan of this paper is the following: All the basic results and definitions required in our framework are recapitulated in Section 2. In the third section, the main result on the convergence and its rates is stated and proved in Theorem 3.1 along with the necessary assumptions. In addition, a convergence rate is also established in Theorem 3.2 for the special case of Hölder stability estimates. In Section 4, we give an example where our results on the convergence can be applied. At the end, a few conclusions are made.

\section{Preliminaries}

Definition 2.1. Duality map: Let $U$ be a Banach space and $U^{*}$ be its dual space. The mapping $J_{p}: U \rightarrow 2^{U^{*}}$ of the convex functional $u \rightarrow \frac{1}{p}\|u\|^{p}$ defined by

$$
J_{p}(u)=\left\{u^{*} \in U^{*} \mid\left\langle u, u^{*}\right\rangle=\|u\|\left\|u^{*}\right\|,\left\|u^{*}\right\|=\|u\|^{p-1}\right\}
$$

is known as the duality mapping of $U$ with the gauge function $t \rightarrow t^{p-1}$, where $p>1$. 
Example 2.1. Let $a>1$. Then, for $U=\mathcal{L}^{a}\left(\mathbb{R}^{n}\right)$ (the space of measurable functions for which the a-th power of the absolute value is Lebesgue integrable), we have

$$
J_{p}: \mathcal{L}^{a}\left(\mathbb{R}^{n}\right) \rightarrow \mathcal{L}^{b}\left(\mathbb{R}^{n}\right) \quad \text { defined by } \quad u(x) \mapsto\|u\|_{U}^{p-a}|u(x)|^{a-2} u(x),
$$

where $a$ and $b$ are conjugate indices.

In general, $J_{p}$ is a set valued mapping but we need it to be single-valued in the further analysis. In order to fulfil this condition, we introduce the notions of uniform convexity and uniform smoothness of Banach spaces.

Definition 2.2. Convexity modulus of $U:$ It is a function $\delta:[0,2] \rightarrow[0,1]$ defined by

$$
\delta_{U}(\epsilon)=\inf \left\{\frac{1}{2}\left(2-\left\|u_{1}+u_{2}\right\|\right): u_{1}, u_{2} \in S,\left\|u_{1}-u_{2}\right\| \geq \epsilon\right\}
$$

where $S$ is the boundary of unit sphere in the Banach space $U$. Further, if $\delta_{U}(\epsilon)>0$ for any $\epsilon \in(0,2]$, then $U$ is uniformly convex.

Definition 2.3. Smoothness modulus of $U$ : It is a function $\rho:[0, \infty) \rightarrow[0, \infty)$ defined by

$$
\rho_{U}(\tau)=\sup \left\{\frac{1}{2}\left(\left\|u_{1}+\tau u_{2}\right\|+\left\|u_{1}-\tau u_{2}\right\|-2\right): u_{1}, u_{2} \in S\right\},
$$

where $S$ is the boundary of unit sphere in the Banach space $U$. Further, if $\lim _{\tau \rightarrow 0} \frac{\rho_{U}(\tau)}{\tau}=0$, then $U$ is uniformly smooth.

Definition 2.4. A Banach space $U$ is

(1) $p$ convex or convex of power type $p$ if $\delta_{U}(\epsilon) \geq Y \epsilon^{p}$, where $Y>0$ is a constant.

(2) $q$ smooth if $\rho_{U}(\tau) \leq Z \tau^{q}$, where $Z>0$ is a constant.

Example 2.2. The Banach space $U=\mathcal{L}^{p}(\Sigma)$, where $p>1$ and $\Sigma \subset \mathbb{R}^{n}$ be an open domain, is uniformly convex as well as uniformly smooth and

$$
\delta_{U}(\epsilon)=\left\{\begin{array}{ll}
\epsilon^{2}, & 1<p<2 \\
\epsilon^{p}, & 2 \leq p<\infty
\end{array} \quad \text { and } \quad \rho_{U}(\tau)= \begin{cases}\tau^{p}, & 1<p<2 \\
\tau^{2}, & 2 \leq p<\infty\end{cases}\right.
$$

Next, we recall the definition of Bregman distance, see [33, Definition 2.56].

Definition 2.5. Bregman distance: Let $U$ be a uniformly smooth Banach space and $J_{p}$ is the duality mapping from $U$ to $U^{*}$ with the gauge function $t \rightarrow t^{p-1}$. Then the functional

$$
\Delta_{p}\left(u_{1}, u_{2}\right)=\frac{1}{p}\left\|u_{1}\right\|^{p}-\frac{1}{p}\left\|u_{2}\right\|^{p}-\left\langle J_{p}\left(u_{2}\right), u_{1}-u_{2}\right\rangle, \quad u_{1} \in U
$$

is the Bregman distance of the convex functional $u \rightarrow \frac{1}{p}\|u\|^{p}$ at $u_{2} \in U$.

The following identity in Lemma 2.1 is known as three point identity for Bregman distances, for proof see [33, Lemma 2.62]. 
Lemma 2.1. For $u_{1}, u_{2}$ and $u_{3}$ in the Banach space $U$, we have

$$
\Delta_{p}\left(u_{1}, u_{2}\right)=\Delta_{p}\left(u_{1}, u_{3}\right)+\Delta_{p}\left(u_{3}, u_{2}\right)+\left\langle J_{p}\left(u_{3}\right)-J_{p}\left(u_{2}\right), u_{1}-u_{3}\right\rangle .
$$

2.1. Iteratively Regularized Landweber Iteration Method. In Banach spaces, we consider the following iteratively regularized Landweber iteration method given in [33]:

$$
\begin{gathered}
J_{p}\left(u_{k+1}^{\delta}-u_{0}\right)=\left(1-\beta_{k}\right) J_{p}\left(u_{k}^{\delta}-u_{0}\right)-\mu F^{\prime}\left(u_{k}^{\delta}\right)^{*} j_{p}\left(F\left(u_{k}^{\delta}\right)-v^{\delta}\right), \\
u_{k+1}^{\delta}=u_{0}+J_{q}^{*}\left(J_{p}\left(u_{k+1}^{\delta}-u_{0}\right)\right), \text { where } 0<\beta_{k} \leq \beta_{\max }<1, k=0,1,2, \cdots
\end{gathered}
$$

Here $J_{p}: U \rightarrow U^{*}, J_{q}^{*}: U^{*} \rightarrow U, j_{p}: V \rightarrow V^{*}$ are duality mappings, $\mu$ is a positive constant, $u_{0}=u_{0}^{\delta}$ is the initial guess of the solution, $v^{\delta} \in V$ be such that $\left\|v^{\delta}-v\right\| \leq \delta$ and $p, q>1$ are conjugate indices. This iterative scheme is a Gradient type method resulting from the application of gradient descent to the misfit $\|F(u)-v\|^{p}$.

Remark 2.1. For Hilbert space settings, convergence of Iteratively regularized Landweber iteration scheme (2.1)-(2.2) has been shown in [25] for the noisy data and the appropriate choice of $\beta_{i}$ 's in $[0,1]$. Also convergence rates have been obtained in [15] provided the exact solution satisfies the source conditions [16]. In [33, Theorem 7.5], convergence rates have been obtained for the method (2.1)-(2.2) in Banach spaces by incorporating the following variational inequalities

$$
\left|\left\langle J_{p}\left(u^{\dagger}-u_{0}\right), u-u^{\dagger}\right\rangle\right| \leq \beta \Delta_{p}^{u_{0}}\left(u^{\dagger}, u\right)^{\frac{1-\nu}{2}}\left\|F^{\prime}\left(u^{\dagger}\right)\left(u-u^{\dagger}\right)\right\|^{\nu},
$$

and the non-linearity estimate

$$
\left\|\left(F^{\prime}\left(u^{\dagger}+v\right)-F^{\prime}\left(u^{\dagger}\right)\right) v\right\| \leq K\left\|F^{\prime}\left(u^{\dagger}\right) v\right\|^{c_{1}} \Delta_{p}^{u_{0}}\left(u^{\dagger}, v+u^{\dagger}\right)^{c_{2}},
$$

where $v \in U$ and $u, u^{\dagger}+v$ are in some ball of positive radius around the exact solution $u^{\dagger}$, $\nu \in(0,1], \beta>0, K>0, \Delta_{p}^{u_{0}}\left(u^{\dagger}, u\right)=\Delta_{p}\left(u^{\dagger}-u_{0}, u-u_{0}\right)$ and $c_{1}, c_{2}$ are properly chosen constants. Here, we study both the convergence and convergence rates by incorporating an alternative condition, namely Hölder type stability (3.2) replacing the variational inequalities and the non-linearity estimate.

Remark 2.2. For solving $F(u)=v$, suppose $v^{\delta}$ is known to us such that $\left\|v^{\delta}-v\right\| \leq \delta$ for some $\delta>0$. Then, consider the following iteration scheme:

$$
\begin{gathered}
J_{p}\left(u_{k+1}^{\delta}\right)=J_{p}\left(u_{k}^{\delta}\right)-\mu F^{\prime}\left(u_{k}^{\delta}\right)^{*} j_{p}\left(F\left(u_{k}^{\delta}\right)-v^{\delta}\right)+\beta_{k} J_{p}\left(u_{0}-u_{k}^{\delta}\right), \\
u_{k+1}^{\delta}=J_{q}^{*}\left(J_{p}\left(u_{k+1}^{\delta}\right)\right), \quad \text { where } \quad 0<\beta_{k} \leq \beta_{\max }<\frac{1}{2} .
\end{gathered}
$$

This is another version of Iteratively regularized Landweber Iteration method. In Hilbert spaces, this method reduces to the method discussed in [4] with $\mu=1$.

Remark 2.3. If $\beta_{k}=0$ for each $k$ in (2.1), then the resulting method is nothing but the Landweber iteration method discussed in [24] with $u_{0}=\delta=0$. 
Now, we recall the properties of duality mappings through which one get to know about the conditions under which the duality mapping $J_{p}$ is single valued, invertible etc., see $[10,17]$.

Theorem 2.1. For $p>1$, the following holds:

(1) For every $u \in U$, the set $J_{p}(u)$ is non empty.

(2) The set $J_{p}(u)$ is single valued for each $u \in U$ provided the Banach space $U$ is uniformly smooth.

(3) If a Banach space is uniformly convex and uniformly smooth, then $J_{p}(u)$ is one-one and onto and its inverse is $J_{p}^{-1}=J_{q}^{*}$, with $J_{q}^{*}$ is the duality mapping of $U^{*}$, where $p, q>1$ with $\frac{1}{p}+\frac{1}{q}=1$ and the associated gauge function is $t \rightarrow t^{q-1}$.

(4) Uniform smoothness (uniform convexity) of a Banach space $U$ is equivalent to the uniform convexity (uniform smoothness) of the dual space $U^{*}$.

Next result recapitulates the main facts of Bregman distance and its relationship with the norm. See [33, Theorem 2.60] for proof of parts (1) and (4) in the following theorem.

Theorem 2.2. Let $U$ be a uniformly convex and uniformly smooth Banach space. Then, for all $u_{1}, u_{2} \in U$, following result holds:

(1) $\Delta_{p}\left(u_{1}, u_{2}\right) \geq 0$ and $\Delta_{p}\left(u_{1}, u_{2}\right)=0$ if and only if $u_{1}=u_{2}$.

(2) If $U$ is $p$ convex, then we have

$$
\Delta_{p}\left(u_{1}, u_{2}\right) \geq \frac{C_{p}}{p}\left\|u_{1}-u_{2}\right\|^{p}
$$

where $C_{p}>0$ is some constant.

(3) If $U^{*}$ is $q$ smooth, then we have

$$
\Delta_{q}\left(u_{1}^{*}, u_{2}^{*}\right) \leq \frac{G_{q}}{q}\left\|u_{1}^{*}-u_{2}^{*}\right\|^{q}, \quad \forall u_{1}^{*}, u_{2}^{*} \in U^{*}
$$

where $G_{q}>0$ is some constant.

(4) Following are equivalent:

(a) $\lim _{n \rightarrow \infty}\left\|u_{n}-u\right\|=0$,

(b) $\lim _{n \rightarrow \infty} \Delta_{p}\left(u_{n}, u\right)=0$ and

(c) $\lim _{n \rightarrow \infty}\left\|u_{n}\right\|=\|u\|$ and $\lim _{n \rightarrow \infty}\left\langle J_{p}\left(u_{n}\right), u\right\rangle=\left\langle J_{p}(u), u\right\rangle$.

Proof of parts (2) and (3) of Theorem 2.2 are discussed after the Remark 2.4. In [24, Theorem 2.5], results of the type (2) and (3) are discussed with the following Bregman distance

$$
\Delta_{p}^{\prime}\left(u_{1}, u_{2}\right)=\frac{1}{p}\left\|u_{2}\right\|^{p}-\frac{1}{p}\left\|u_{1}\right\|^{p}-\left\langle J_{p}\left(u_{1}\right), u_{2}-u_{1}\right\rangle, \quad u_{1}, u_{2} \in U .
$$

Note that the definitions of Bregman distance employed in [24] and in this paper are different, because of the interchange of arguments. 
Remark 2.4. [38, Theorem 1] Let $\delta_{X}(\epsilon)$ represents the convexity modulus of a uniformly convex real Banach space $X$. Then, there exists a function $\phi_{p} \in \mathbb{A}$ such that

$$
\left\|x_{1}+x_{2}\right\|^{p} \geq\left\|x_{1}\right\|^{p}+p\left\langle J_{p}\left(x_{1}\right), x_{2}\right\rangle+\sigma_{p}\left(x_{1}, x_{2}\right), \quad x_{1}, x_{2} \in X
$$

where

$$
\sigma_{p}\left(x_{1}, x_{2}\right)=p \int_{0}^{1} \frac{\left(\left\|x_{1}+t x_{2}\right\| \vee\left\|x_{1}\right\|\right)^{p}}{t} \phi_{p}\left(\frac{t\left\|x_{2}\right\|}{\left\|x_{1}+t x_{2}\right\| \vee\left\|x_{1}\right\|}\right) d t
$$

(see Remark 2.5) and

$$
\begin{gathered}
\mathbb{A}=\left\{\phi: \mathbb{R}^{+} \rightarrow \mathbb{R}^{+}: \phi(0)=0, \phi(t) \text { is strictly increasing and } K\right. \text { is a positive } \\
\text { constant such that } \left.\phi(t) \geq K \delta_{X}(t / 2)\right\} .
\end{gathered}
$$

Here $x \wedge y=\min (x, y)$ and $x \vee y=\max (x, y)$ for arbitrarily real numbers $x$ and $y$. Since, $\phi_{p} \in \mathbb{A},(2.6)$ can be written as

$$
\sigma_{p}\left(x_{1}, x_{2}\right) \geq p K_{p} \int_{0}^{1} \frac{\left(\left\|x_{1}+t x_{2}\right\| \vee\left\|x_{1}\right\|\right)^{p}}{t} \delta_{X}\left(\frac{t\left\|x_{2}\right\|}{2\left(\left\|x_{1}+t x_{2}\right\| \vee\left\|x_{1}\right\|\right)}\right) d t
$$

with

$$
\begin{aligned}
& K_{p}=4(2+\sqrt{3}) \min \left\{\frac{1}{2} p(p-1) \wedge 1,\left(\frac{1}{2} p \wedge 1\right)(p-1),\right. \\
&\left.(p-1)\left[1-(\sqrt{3}-1)^{q}\right), 1-\left[1+\frac{(2-\sqrt{3}) p}{p-1}\right]^{1-p}\right\},
\end{aligned}
$$

where the value of $K_{p}$ is obtained from Lemma 3 in [38]. Also if $X$ is $p$ convex, then last inequality can be written as

$$
\begin{gathered}
\sigma_{p}\left(x_{1}, x_{2}\right) \geq p Y K_{p} \int_{0}^{1} \frac{\left(\left\|x_{1}+t x_{2}\right\| \vee\left\|x_{1}\right\|\right)^{p}}{t}\left(\frac{t\left\|x_{2}\right\|}{2\left(\left\|x_{1}+t x_{2}\right\| \vee\left\|x_{1}\right\|\right)}\right)^{p} d t \\
=p\left(\frac{Y K_{p}}{2^{p}}\right)\left\|x_{2}\right\|^{p} \int_{0}^{1} t^{p-1} d t=C_{p}\left\|x_{2}\right\|^{p}
\end{gathered}
$$

for some positive constants $Y$ and $C_{p}=\frac{Y K_{p}}{2^{p}}$. Above inequality and (2.5) imply that

$$
\frac{1}{p}\left\|x_{1}+x_{2}\right\|^{p}-\frac{1}{p}\left\|x_{1}\right\|^{p}-\left\langle J_{p}\left(x_{1}\right), x_{2}\right\rangle \geq \frac{1}{p} \sigma_{p}\left(x_{1}, x_{2}\right) \geq \frac{C_{p}}{p}\left\|x_{2}\right\|^{p} .
$$

Now we come to the proof of part (2) of Theorem 2.2. In our notations, if we consider $X=U$, $x_{1}=u_{2}$ and $x_{2}=u_{1}-u_{2}$, then (2.8) implies that

$$
\Delta_{p}\left(u_{1}, u_{2}\right)=\frac{1}{p}\left\|u_{1}\right\|^{p}-\frac{1}{p}\left\|u_{2}\right\|^{p}-\left\langle J_{p}\left(u_{2}\right), u_{1}-u_{2}\right\rangle \geq \frac{C_{p}}{p}\left\|u_{1}-u_{2}\right\|^{p},
$$

which is the desired inequality. Here $C_{p}$ is a constant depending on $p$. Part (3) can be proved similarly by using Theorem 2 in [38]. 
Remark 2.5. In [38, equation 2.2], value of $\sigma_{p}$ given in the statement is

$$
\sigma_{p}(x, y)=p \int_{0}^{1} \frac{(\|x+t y\| \vee\|x\|)^{p}}{t} \phi_{p}\left(\frac{t\|y\|}{(\|x+t y\| \vee\|y\|)}\right) d t .
$$

But the actual value is

$$
\sigma_{p}(x, y)=p \int_{0}^{1} \frac{(\|x+t y\| \vee\|x\|)^{p}}{t} \phi_{p}\left(\frac{t\|y\|}{(\|x+t y\| \vee\|x\|)}\right) d t
$$

which can be easily verified from the proof given there.

\section{Convergence and Convergence Rates}

In the present section, we analyze the convergence and its rates for the iteratively regularized Landweber iteration method (2.1)-(2.2). Here, we consider the notation

$$
B=B_{\rho}^{\Delta}\left(u^{\dagger}\right):=\left\{u \in U: \Delta_{p}^{u_{0}}\left(u^{\dagger}, u\right) \leq \rho^{2}\right\}
$$

where $\Delta_{p}^{u_{0}}\left(u^{\dagger}, u\right)=\Delta_{p}\left(u^{\dagger}-u_{0}, u-u_{0}\right), \rho>0$ is some constant and $u^{\dagger}$ is the solution of $F(u)=v$ which may not be unique. We assume $B \subset D(F)$. To prove the main results of the paper, we need to have certain assumptions accumulated below.

\section{Assumption 3.1.}

(1) $U$ is $q$ smooth and $p$ convex with $\frac{1}{p}+\frac{1}{q}=1$, where $p, q>1$.

(2) $F$ has a Fréchet derivative $F^{\prime}(\cdot)$ and it satisfies the following local estimate

$$
\left\|F^{\prime}\left(u_{1}\right)-F^{\prime}\left(u_{2}\right)\right\| \leq L\left\|u_{1}-u_{2}\right\|, \quad \forall u_{1}, u_{2} \in B
$$

where $L>0$ is a constant.

(3) $F^{\prime}(\cdot)$ satisfies the boundedness condition, i.e. $\left\|F^{\prime}(u)\right\| \leq \hat{L}$ for all $u \in B$ for some positive constant $\hat{L}$.

(4) F is weakly sequentially closed.

(5) Elements in B satisfy the following Hölder stability estimate

$$
\Delta_{p}^{u_{0}}\left(u_{1}, u_{2}\right) \leq C_{F}^{p}\left\|F\left(u_{1}\right)-F\left(u_{2}\right)\right\|^{\frac{1+\epsilon}{2} p}, \quad u_{1}, u_{2} \in B, \epsilon \in(0,1],
$$

where $C_{F}>0$ is a constant.

(6) $u_{0}$ lies in $B$ and there exists a sequence $\left\{r_{k}\right\}_{k \in \mathbb{N} \cup\{0\}}$ such that $\Delta_{p}^{u_{0}}\left(u_{k}^{\delta}, u^{\dagger}\right) \geq r_{k}\left(\Delta_{p}^{u_{0}}\left(u^{\dagger}, u_{k}^{\delta}\right)\right)$ for each $k$. For example in Hilbert spaces, we have

$$
\Delta_{2}^{u_{0}}\left(u^{\dagger}, u_{k}^{\delta}\right)=\frac{1}{2}\left\|u^{\dagger}-u_{k}^{\delta}\right\|^{2}=\Delta_{2}^{u_{0}}\left(u_{k}^{\delta}, u^{\dagger}\right)
$$

which means $r_{k}=1$ for each $k$.

(7) The sequence $\left\{\beta_{k}\right\}$ satisfies (2.2), $\sum_{k} \beta_{k}<\infty$ and $\beta_{\max }$ is sufficiently small.

(8) $\mu$ is chosen such that

$$
\mu^{q-1}<\frac{q}{2^{q} \hat{L}^{q} G_{q}}
$$


(9) $\rho^{2}$ satisfies

$$
\rho^{2}=\hat{L}^{-p}\left(L C_{F}^{2}\right)^{\frac{-p}{\epsilon}}\left(\frac{C_{p}}{p}\right)^{1+\frac{2}{\epsilon}} .
$$

(10) a-priori choice of the stopping index $k_{\star}$ is

$$
k_{\star}(\delta)=\min \left\{k \in \mathbb{N}: \beta_{k} \leq \tau \delta\right\},
$$

with $\tau>0$ sufficiently large.

Remark 3.1. The Hölder type stability estimate (3.2) for the special case $p=2$ can be obtained by a lower bound on the Fréchet derivative $F^{\prime}$. Let there exists a constant $K>0$ such that

$$
\left\|F^{\prime}(u)\left(\frac{u-u^{\dagger}}{\left\|u-u^{\dagger}\right\|}\right)\right\| \geq K\left\|u-u^{\dagger}\right\|^{1-\epsilon_{1}} \quad \forall u \in D(F) \cap B_{r}\left(u^{\dagger}\right),
$$

where $B_{r}\left(u^{\dagger}\right)$ is some ball of radius $r$ (sufficiently small) around $u^{\dagger}$ and $\epsilon_{1} \in(0,1]$. The last inequality and the estimate

$$
\left\|F\left(u^{\prime}\right)-F(u)-F^{\prime}(u)\left(u^{\prime}-u\right)\right\| \leq \frac{L}{2}\left\|u^{\prime}-u\right\|^{2} \quad \forall u, u^{\prime} \in D(F),
$$

imply that

$$
\begin{gathered}
K\left\|u-u^{\dagger}\right\|^{2-\epsilon_{1}} \leq\left\|F(u)-F\left(u^{\dagger}\right)-F^{\prime}(u)\left(u-u^{\dagger}\right)\right\|+\left\|F(u)-F\left(u^{\dagger}\right)\right\| \\
\leq \frac{L}{2}\left\|u-u^{\dagger}\right\|^{2}+\left\|F(u)-F\left(u^{\dagger}\right)\right\|, \quad \forall u \in D(F) \cap B_{r}\left(u^{\dagger}\right) .
\end{gathered}
$$

Since $r$ is small, last inequality can also be written as

$$
K\left\|u-u^{\dagger}\right\|^{2-\epsilon_{1}} \leq \frac{L}{2}\left\|u-u^{\dagger}\right\|^{2-\epsilon_{1}}+\left\|F(u)-F\left(u^{\dagger}\right)\right\|
$$

which immediately leads to the estimate

$$
\left\|u-u^{\dagger}\right\| \leq C^{\prime}\left\|F(u)-F\left(u^{\dagger}\right)\right\|^{\frac{1}{2-\epsilon_{1}}} \quad \forall u \in D(F) \cap B_{r}\left(u^{\dagger}\right),
$$

where $C^{\prime}$ is a constant depending on $K$ and $L$. Since, in the case of Hilbert spaces, $p=2$ and $\Delta_{2}^{u_{0}}\left(u, u^{\dagger}\right)=\frac{1}{2}\left\|u-u^{\dagger}\right\|^{2}$ where $u \in U$, an estimate of the type (3.2) can be obtained. In general, it is impossible to obtain a lower bound for $F^{\prime}$ due to ill-posedness of almost all the inverse problems. This lower bound has been studied for many inverse problems under various assumptions, see, for instance, $[7,13]$. The key fact used in $[7,13]$ to obtain the lower bounds is that the forward operator has been projected properly.

Remark 3.2. For $\epsilon=1$ and $u_{0}=0$ in (3.2) (observe that (3.2) with $\epsilon=1$ is the Lipschitz-type stability estimate), we have

$$
\begin{aligned}
\left\langle J_{p}\left(u^{\dagger}\right), u-u^{\dagger}\right\rangle & \leq\left\|u^{\dagger}\right\|^{p-1}\left\|u-u^{\dagger}\right\| \leq\left(\frac{p}{C_{p}}\right)^{\frac{1}{p}}\left\|u^{\dagger}\right\|^{p-1} \Delta_{p}\left(u, u^{\dagger}\right)^{\frac{1}{p}} \\
& \leq K\left\|F(u)-F\left(u^{\dagger}\right)\right\| \quad \forall u \in B,
\end{aligned}
$$

where above holds by using (2.3) and $K=C_{F}\left\|u^{\dagger}\right\|^{p-1}\left(\frac{p}{C_{p}}\right)^{\frac{1}{p}}$. In [26], it is shown that the last inequality implies the source condition $J_{p}\left(u^{\dagger}\right)=F^{\prime}\left(u^{\dagger}\right)^{*} v$ for some $v$ such that $\|v\| \leq 1$. 
Now, we are ready to state our main result in which we obtain the convergence and its rates with some additional assumptions on the sequence $\left\{\beta_{k}\right\}$.

Theorem 3.1. Let $F$ be a non-linear operator between the Banach spaces $U, V$ and the operator equation $F(u)=v, v \in V$, has a solution $u^{\dagger}$. Suppose that the Assumption 3.1 holds, $v^{\delta} \in V$ be such that $\left\|v^{\delta}-v\right\| \leq \delta$. Then all the iterates $u_{k+1}^{\delta}$ of iteratively regularized Landweber iteration method (2.1)-(2.2) remain in $B$ for all $k \leq k_{\star}(\delta)-1$ provided $\beta_{\max }$ is sufficiently small (see Lemma 3.2 for exact estimate of $\left.\beta_{\max }\right)$. Moreover, iterates satisfy the recurrence relation

$$
\gamma_{k+1}^{\delta} \leq \gamma_{k}^{\delta}+K_{1} \delta^{p}+K_{2} \delta^{\epsilon}+K_{3} \delta^{p+\epsilon}+K_{4} \delta-K_{6} \rho^{2},
$$

for some constants $K_{i}, 1 \leq i \leq 4$, and $K_{6}>0$, where $\gamma_{k}=\Delta_{p}^{u_{0}}\left(u^{\dagger}, u_{k}^{\delta}\right)$. We also obtain the convergence rates

$$
\Delta_{p}^{u_{0}}\left(u^{\dagger}, u_{k_{\star}}\right)-\left(1-K_{6}\right) \rho^{2}=O\left(\delta^{\epsilon}\right), \text { as } \delta \rightarrow 0 .
$$

For $\delta=0$, iterates $u_{k+1}$ of iteratively regularized Landweber iteration method (2.1)-(2.2) not only remain in $B$ but also converge to the solution $u^{\dagger}$. Further, we get the following rates:

(1) Iterates $\gamma_{k}=\Delta_{p}^{u_{0}}\left(u^{\dagger}, u_{k}\right)$ satisfy the recursion formula

$$
\gamma_{k+1} \leq-K_{8} \gamma_{k}^{\frac{2}{1+\epsilon}}+\alpha_{k} \gamma_{k}+K_{11} \beta_{k}
$$

for some positive constants $K_{8}, K_{11}$ and $\left\{\alpha_{k}\right\}$ is a sequence converges to 1 . Further, if $\left\{\beta_{k}\right\}$ satisfies $\beta_{k} \leq C \gamma_{k}$ (smoothness condition) for some constant $C>0$, then the convergence rate, for $\epsilon \in(0,1)$, is given by

$$
\Delta_{p}^{u_{0}}\left(u^{\dagger}, u_{k}\right) \leq\left(\left(g_{k} \rho^{2}\right)^{-\frac{1-\epsilon}{1+\epsilon}}+h_{k}\right)^{-\frac{1+\epsilon}{1-\epsilon}}, \quad k=1,2, \ldots
$$

where

$$
g_{k}=\prod_{i=0}^{k-1} d_{i}, k \geq 1, \text { and } h_{k}=\sum_{j=1}^{k-1}\left(d_{j} d_{j+1} \ldots d_{k-1}\right)^{-\frac{1+\epsilon}{1-\epsilon}} f_{j-1}+f_{k-1}, k \geq 2, h_{1}=f_{0}
$$

with $f_{k}=t e_{k} d_{k}^{-t}, d_{k}=\alpha_{k}+C K_{11}, e_{k}=\frac{K_{8}}{d_{k}}$ and $t=\frac{1-\epsilon}{1+\epsilon}$.

For $\epsilon=1$, we get

$$
\Delta_{p}^{u_{0}}\left(u^{\dagger}, u_{k}\right) \leq \prod_{i=0}^{k-1}\left(-K_{8}+\alpha_{i}+K_{11} C\right) \rho^{2}, \quad k=1,2, \ldots
$$

(2) we also obtain the rate

$$
\Delta_{p}^{u_{0}}\left(u^{\dagger}, u_{k}\right)=O\left(\beta_{k}^{q-1}\right), \quad \text { as } k \rightarrow \infty
$$

provided

$$
K_{12}+\eta \beta_{k}^{-1}\left[\alpha_{k}-\left(\frac{\beta_{k+1}}{\beta_{k}}\right)^{q-1}\right] \leq 0
$$

for some constants $\eta, K_{12}$, and a sequence $\left\{\alpha_{k}\right\}$ converging to 1 . 
Instead of giving a single proof of Theorem 3.1, we discuss it in parts in the form of a series of lemmas to have a better understanding. In the first lemma, we obtain an estimate of $\Delta_{p}^{u_{0}}\left(u^{\dagger}, u_{k+1}^{\delta}\right)-\Delta_{p}^{u_{0}}\left(u^{\dagger}, u_{k}^{\delta}\right)$.

Lemma 3.1. Let $F$ be a non-linear operator between the Banach spaces $U, V$ and the operator equation $F(u)=v, v \in V$, has a solution $u^{\dagger}$. Suppose that Assumption 3.1 holds and $v^{\delta} \in V$ be such that $\left\|v^{\delta}-v\right\| \leq \delta$. Then the iterates $u_{k+1}^{\delta}$ of iteratively regularized Landweber iteration method (2.1)-(2.2) satisfy the following inequality

$$
\begin{gathered}
\Delta_{p}^{u_{0}}\left(u^{\dagger}, u_{k+1}^{\delta}\right)-\Delta_{p}^{u_{0}}\left(u^{\dagger}, u_{k}^{\delta}\right) \leq\left(2^{q-1} \frac{G_{q}}{q} \mu^{q} \hat{L}^{q}-\mu\right)\left\|F\left(u_{k}^{\delta}\right)-v^{\delta}\right\|^{p}+\frac{\mu}{2} L C_{F}^{2}\left(\frac{p}{C_{p}}\right)^{2 / p}\left\|F\left(u_{k}^{\delta}\right)-v^{\delta}\right\|^{p+\epsilon} \\
+\left(2^{p+q-2} \frac{G_{q}}{q} \beta_{k}^{q}+\beta_{k} \frac{(p-1) \epsilon_{2}^{\frac{p}{p-1}}}{p}\right)\left\|u^{\dagger}-u_{0}\right\|^{p}+\mu\left\|F\left(u_{k}^{\delta}\right)-v^{\delta}\right\|^{p-1} \delta+\left(\frac{\beta_{k} \epsilon_{2}^{-p}}{C_{p}}-\right. \\
\left.\left(1+r_{k}\right) \beta_{k}+2^{p+q-2} \beta_{k}^{q} \frac{G_{q}}{q} \frac{p}{C_{p}}\right) \gamma_{k}^{\delta}, \quad \text { where } \epsilon_{2}>0 .
\end{gathered}
$$

Proof. From Lemma 2.1 and (2.1), we can write

$$
\begin{gathered}
\Delta_{p}^{u_{0}}\left(u^{\dagger}, u_{k+1}^{\delta}\right)-\Delta_{p}^{u_{0}}\left(u^{\dagger}, u_{k}^{\delta}\right)=\Delta_{p}^{u_{0}}\left(u_{k}^{\delta}, u_{k+1}^{\delta}\right)+\left\langle J_{p}\left(u_{k}^{\delta}-u_{0}\right)-J_{p}\left(u_{k+1}^{\delta}-u_{0}\right), u^{\dagger}-u_{k}^{\delta}\right\rangle \\
=\Delta_{p}^{u_{0}}\left(u_{k}^{\delta}, u_{k+1}^{\delta}\right)-\mu\left\langle j_{p}\left(F\left(u_{k}^{\delta}\right)-v^{\delta}\right), F^{\prime}\left(u_{k}^{\delta}\right)\left(u_{k}^{\delta}-u^{\dagger}\right)\right\rangle+\beta_{k}\left\langle J_{p}\left(u^{\dagger}-u_{0}\right), u^{\dagger}-u_{k}^{\delta}\right\rangle \\
-\beta_{k}\left\langle J_{p}\left(u^{\dagger}-u_{0}\right)-J_{p}\left(u_{k}^{\delta}-u_{0}\right), u^{\dagger}-u_{k}^{\delta}\right\rangle .
\end{gathered}
$$

Now, we estimate each of the four terms of the right side to (3.6) individually. For the first term, using Definitions 2.5 and 2.1, we have

$$
\begin{gathered}
\Delta_{p}^{u_{0}}\left(u_{k}^{\delta}, u_{k+1}^{\delta}\right)=\frac{1}{p}\left\|u_{k}^{\delta}-u_{0}\right\|^{p}-\frac{1}{p}\left\|u_{k+1}^{\delta}-u_{0}\right\|^{p}-\left\langle J_{p}\left(u_{k+1}^{\delta}-u_{0}\right), u_{k}^{\delta}-u_{k+1}^{\delta}\right\rangle \\
=\frac{1}{q}\left\|u_{k+1}^{\delta}-u_{0}\right\|^{p}-\frac{1}{q}\left\|u_{k}^{\delta}-u_{0}\right\|^{p}-\left\langle J_{p}\left(u_{k+1}^{\delta}-u_{0}\right), u_{k}^{\delta}-u_{k+1}^{\delta}\right\rangle+\left\|u_{k}^{\delta}-u_{0}\right\|^{p}-\left\|u_{k+1}^{\delta}-u_{0}\right\|^{p} \\
=\frac{1}{q}\left\|u_{k+1}^{\delta}-u_{0}\right\|^{p}-\frac{1}{q}\left\|u_{k}^{\delta}-u_{0}\right\|^{p}-\left\langle J_{p}\left(u_{k+1}^{\delta}-u_{0}\right),\left(u_{k}^{\delta}-u_{0}\right)-\left(u_{k+1}^{\delta}-u_{0}\right)\right\rangle \\
+\left\langle u_{k}^{\delta}-u_{0}, J_{p}\left(u_{k}^{\delta}-u_{0}\right)\right\rangle-\left\langle u_{k+1}^{\delta}-u_{0}, J_{p}\left(u_{k+1}^{\delta}-u_{0}\right)\right\rangle \\
=\frac{1}{q}\left\|u_{k+1}^{\delta}-u_{0}\right\|^{p}-\frac{1}{q}\left\|u_{k}^{\delta}-u_{0}\right\|^{p}+\left\langle u_{k}^{\delta}-u_{0}, J_{p}\left(u_{k}^{\delta}-u_{0}\right)-J_{p}\left(u_{k+1}^{\delta}-u_{0}\right)\right\rangle \\
=\frac{1}{q}\left\|J_{p}\left(u_{k+1}^{\delta}-u_{0}\right)\right\|^{q}-\frac{1}{q}\left\|J_{p}\left(u_{k}^{\delta}-u_{0}\right)\right\|^{q}-\left\langle u_{k}^{\delta}-u_{0}, J_{p}\left(u_{k+1}^{\delta}-u_{0}\right)-J_{p}\left(u_{k}^{\delta}-u_{0}\right)\right\rangle \\
=\Delta_{q}\left(J_{p}\left(u_{k+1}^{\delta}-u_{0}\right), J_{p}\left(u_{k}^{\delta}-u_{0}\right)\right) .
\end{gathered}
$$

Use (2.4) and then (2.1) in above to obtain

$$
\begin{aligned}
\Delta_{p}^{u_{0}}\left(u_{k}^{\delta}, u_{k+1}^{\delta}\right) & \leq \frac{G_{q}}{q}\left\|J_{p}\left(u_{k+1}^{\delta}-u_{0}\right)-J_{p}\left(u_{k}^{\delta}-u_{0}\right)\right\|^{q} \\
& =\frac{G_{q}}{q}\left\|\beta_{k} J_{p}\left(u_{k}^{\delta}-u_{0}\right)+\mu F^{\prime}\left(u_{k}^{\delta}\right)^{*} j_{p}\left(F\left(u_{k}^{\delta}\right)-v^{\delta}\right)\right\|^{q} .
\end{aligned}
$$


Now using the estimate

$$
\left\|u_{1}+u_{2}\right\|^{r} \leq 2^{r-1}\left(\left\|u_{1}\right\|^{r}+\left\|u_{2}\right\|^{r}\right), \quad r \geq 1, \quad u_{1}, u_{2} \in U
$$

see [26, Lemma 3.20], twice into (3.7), we have

$$
\begin{gathered}
\Delta_{p}^{u_{0}}\left(u_{k}^{\delta}, u_{k+1}^{\delta}\right) \leq 2^{q-1} \frac{G_{q}}{q}\left(\beta_{k}^{q}\left\|J_{p}\left(u_{k}^{\delta}-u_{0}\right)\right\|^{q}+\mu^{q}\left\|F^{\prime}\left(u_{k}^{\delta}\right)^{*} j_{p}\left(F\left(u_{k}^{\delta}\right)-v^{\delta}\right)\right\|^{q}\right) \\
\quad=2^{q-1} \frac{G_{q}}{q}\left(\beta_{k}^{q}\left\|u_{k}^{\delta}-u_{0}\right\|^{p}+\mu^{q}\left\|F^{\prime}\left(u_{k}^{\delta}\right)^{*} j_{p}\left(F\left(u_{k}^{\delta}\right)-v^{\delta}\right)\right\|^{q}\right) \\
\leq 2^{q-1} \frac{G_{q}}{q}\left(2^{p-1} \beta_{k}^{q}\left(\left\|u^{\dagger}-u_{0}\right\|^{p}+\left\|u^{\dagger}-u_{k}^{\delta}\right\|^{p}\right)+\mu^{q}\left\|F^{\prime}\left(u_{k}^{\delta}\right)^{*} j_{p}\left(F\left(u_{k}^{\delta}\right)-v^{\delta}\right)\right\|^{q}\right) \\
\leq 2^{q-1} \frac{G_{q}}{q}\left(2^{p-1} \beta_{k}^{q}\left(\left\|u^{\dagger}-u_{0}\right\|^{p}+\frac{p}{C_{p}} \Delta_{p}^{u_{0}}\left(u^{\dagger}, u_{k}^{\delta}\right)\right)+\mu^{q} \hat{L}^{q}\left\|F\left(u_{k}^{\delta}\right)-v^{\delta}\right\|^{p}\right),
\end{gathered}
$$

where the last inequality is obtained by incorporating (2.3) and (3) of Assumption 3.1 provided $u_{k}^{\delta}$ satisfies the estimate (3.2) which will be shown later.

Next, let us estimate the second term on the right side to (3.6) as

$$
\begin{aligned}
& -\mu\left\langle j_{p}\left(F\left(u_{k}^{\delta}\right)-v^{\delta}\right), F^{\prime}\left(u_{k}^{\delta}\right)\left(u_{k}^{\delta}-u^{\dagger}\right)\right\rangle \\
& =-\mu\left\langle j_{p}\left(F\left(u_{k}^{\delta}\right)-v^{\delta}\right), F\left(u_{k}^{\delta}\right)-v^{\delta}\right\rangle+\mu\left\langle j_{p}\left(F\left(u_{k}^{\delta}\right)-v^{\delta}\right), F\left(u_{k}^{\delta}\right)-v^{\delta}-F^{\prime}\left(u_{k}^{\delta}\right)\left(u_{k}^{\delta}-u^{\dagger}\right)\right\rangle \\
& \quad=-\mu\left\|F\left(u_{k}^{\delta}\right)-v^{\delta}\right\|^{p}+\mu\left\langle j_{p}\left(F\left(u_{k}^{\delta}\right)-v^{\delta}\right), F\left(u_{k}^{\delta}\right)-v^{\delta}-F^{\prime}\left(u_{k}^{\delta}\right)\left(u_{k}^{\delta}-u^{\dagger}\right)\right\rangle .
\end{aligned}
$$

By employing fundamental theorem of calculus for $F^{\prime}(\cdot)$, i.e.

$$
\left\|F\left(u_{k}^{\delta}\right)-v^{\delta}-F^{\prime}\left(u_{k}^{\delta}\right)\left(u_{k}^{\delta}-u^{\dagger}\right)\right\| \leq \frac{L}{2}\left\|u_{k}^{\delta}-u^{\dagger}\right\|^{2}+\delta,
$$

and (3.1) in the last equality to obtain

$$
\begin{aligned}
& -\mu\left\langle j_{p}\left(F\left(u_{k}^{\delta}\right)-v^{\delta}\right), F^{\prime}\left(u_{k}^{\delta}\right)\left(u_{k}^{\delta}-u^{\dagger}\right)\right\rangle \\
& \quad \leq-\mu\left\|F\left(u_{k}^{\delta}\right)-v^{\delta}\right\|^{p}+\frac{\mu L}{2}\left\|F\left(u_{k}^{\delta}\right)-v^{\delta}\right\|^{p-1}\left\|u_{k}^{\delta}-u^{\dagger}\right\|^{2}+\mu\left\|F\left(u_{k}^{\delta}\right)-v^{\delta}\right\|^{p-1} \delta .
\end{aligned}
$$

Using (2.3) and then (3.2), we further estimate

$$
\begin{aligned}
& -\mu\left\langle j_{p}\left(F\left(u_{k}^{\delta}\right)-v^{\delta}\right), F^{\prime}\left(u_{k}^{\delta}\right)\left(u_{k}^{\delta}-u^{\dagger}\right)\right\rangle \\
& \quad \leq-\mu\left\|F\left(u_{k}^{\delta}\right)-v^{\delta}\right\|^{p}+\frac{\mu}{2} L C_{F}^{2}\left(\frac{p}{C_{p}}\right)^{2 / p}\left\|F\left(u_{k}^{\delta}\right)-v^{\delta}\right\|^{p+\epsilon}+\mu\left\|F\left(u_{k}^{\delta}\right)-v^{\delta}\right\|^{p-1} \delta .
\end{aligned}
$$

Now, let us turn to estimate the third term of the right side to (3.6) as

$$
\begin{aligned}
\beta_{k}\left\langle J_{p}\left(u^{\dagger}-u_{0}\right), u^{\dagger}-u_{k}^{\delta}\right\rangle & \leq \beta_{k}\left|\left\langle J_{p}\left(u^{\dagger}-u_{0}\right), u^{\dagger}-u_{k}^{\delta}\right\rangle\right| \\
& \leq \beta_{k}\left\|J_{p}\left(u^{\dagger}-u_{0}\right)\right\|\left\|u^{\dagger}-u_{k}^{\delta}\right\| \\
& =\beta_{k}\left\|u^{\dagger}-u_{0}\right\|^{p-1}\left\|u^{\dagger}-u_{k}^{\delta}\right\| .
\end{aligned}
$$


Thanks to Young's inequality $a b \leq \frac{a^{r}}{r}+\frac{b^{s}}{s}$ with Hölder conjugates $r, s$ for $a=\epsilon_{2}\left\|u^{\dagger}-u_{0}\right\|^{p-1}$, $b=\epsilon_{2}^{-1}\left\|u^{\dagger}-u_{k}^{\delta}\right\|, r=\frac{p}{p-1}, s=p, \epsilon_{2}>0$, and (2.3) to further yield

$$
\begin{aligned}
\beta_{k}\left\langle J_{p}\left(u^{\dagger}-u_{0}\right), u^{\dagger}-u_{k}^{\delta}\right\rangle & \leq \beta_{k}\left(\frac{(p-1) \epsilon_{2}^{\frac{p}{p-1}}}{p}\left\|u^{\dagger}-u_{0}\right\|^{p}+\frac{\epsilon_{2}^{-p}}{p}\left\|u^{\dagger}-u_{k}^{\delta}\right\|^{p}\right) \\
\leq & \beta_{k}\left(\frac{(p-1) \epsilon_{2}^{\frac{p}{p-1}}}{p}\left\|u^{\dagger}-u_{0}\right\|^{p}+\frac{\epsilon_{2}^{-p}}{C_{p}} \Delta_{p}^{u_{0}}\left(u^{\dagger}, u_{k}^{\delta}\right)\right) .
\end{aligned}
$$

Finally, using Lemma 2.1 in the fourth term on the right hand side to (3.6) to obtain

$$
\begin{gathered}
-\beta_{k}\left\langle J_{p}\left(u^{\dagger}-u_{0}\right)-J_{p}\left(u_{k}^{\delta}-u_{0}\right), u^{\dagger}-u_{k}^{\delta}\right\rangle=-\beta_{k} \Delta_{p}^{u_{0}}\left(u^{\dagger}, u_{k}^{\delta}\right)-\beta_{k} \Delta_{p}^{u_{0}}\left(u_{k}^{\delta}, u^{\dagger}\right)+\beta_{k} \Delta_{p}^{u_{0}}\left(u_{k}^{\delta}, u_{k}^{\delta}\right) \\
\leq-\beta_{k} \Delta_{p}^{u_{0}}\left(u^{\dagger}, u_{k}^{\delta}\right)-\beta_{k} \Delta_{p}^{u_{0}}\left(u_{k}^{\delta}, u^{\dagger}\right) \leq-\beta_{k}\left(1+r_{k}\right) \Delta_{p}^{u_{0}}\left(u^{\dagger}, u_{k}^{\delta}\right)
\end{gathered}
$$

where the last inequality holds because of (6) of Assumption 3.1. Inserting all the estimates (3.8)-(3.11) into (3.6) and use the notation $\gamma_{k}^{\delta}=\Delta_{p}^{u_{0}}\left(u^{\dagger}, u_{k}^{\delta}\right)$, we have

$$
\begin{gathered}
\gamma_{k+1}^{\delta}-\gamma_{k}^{\delta} \leq 2^{q-1} \frac{G_{q}}{q}\left(2^{p-1} \beta_{k}^{q}\left(\left\|u^{\dagger}-u_{0}\right\|^{p}+\frac{p}{C_{p}} \gamma_{k}^{\delta}\right)+\mu^{q} \hat{L}^{q}\left\|F\left(u_{k}^{\delta}\right)-v^{\delta}\right\|^{p}\right)-\mu\left\|F\left(u_{k}^{\delta}\right)-v^{\delta}\right\|^{p} \\
+\frac{\mu}{2} L C_{F}^{2}\left(\frac{p}{C_{p}}\right)^{2 / p}\left\|F\left(u_{k}^{\delta}\right)-v^{\delta}\right\|^{p+\epsilon}+\beta_{k}\left(\frac{(p-1) \epsilon_{2}^{\frac{p}{p-1}}}{p}\left\|u^{\dagger}-u_{0}\right\|^{p}\right)+\frac{\beta_{k} \epsilon_{2}^{-p}}{C_{p}} \gamma_{k}^{\delta} \\
\quad-\beta_{k}\left(1+r_{k}\right) \gamma_{k}^{\delta}+\mu\left\|F\left(u_{k}^{\delta}\right)-v^{\delta}\right\|^{p-1} \delta \\
=\left(2^{q-1} \frac{G_{q}}{q} \mu^{q} \hat{L}^{q}-\mu\right)\left\|F\left(u_{k}^{\delta}\right)-v^{\delta}\right\|^{p}+\left(2^{p+q-2} \frac{G_{q}}{q} \beta_{k}^{q}+\beta_{k} \frac{(p-1) \epsilon_{2}^{\frac{p}{p-1}}}{p}\right)\left\|u^{\dagger}-u_{0}\right\|^{p}+\frac{\mu}{2} L C_{F}^{2}\left(\frac{p}{C_{p}}\right)^{2 / p} \\
\times\left\|F\left(u_{k}^{\delta}\right)-v^{\delta}\right\|^{p+\epsilon}+\left(\frac{\beta_{k} \epsilon_{2}^{-p}}{C_{p}}-\left(1+r_{k}\right) \beta_{k}+2^{p+q-2} \beta_{k}^{q} \frac{G_{q}}{q} \frac{p}{C_{p}}\right) \gamma_{k}^{\delta}+\mu\left\|F\left(u_{k}^{\delta}\right)-v^{\delta}\right\|^{p-1} \delta . \quad(3.12)
\end{gathered}
$$

Remark 3.3. We have intentionally introduced the parameter $\epsilon_{2}$ in (3.10). Rationale behind the introduction of this $\epsilon_{2}$ is discussed in Remark 3.5.

In the next lemma, we show that all the iterates of our iteration scheme remain in $B$ using Lemma 3.1 under certain assumptions.

Lemma 3.2. Suppose that all the assumptions of Lemma 3.1 hold. Then, all the iterates of (2.1)-(2.2) remain in $B$ for all $k \leq k_{*}(\delta)-1$ provided $\tau$ defined in (10) of Assumption 3.1 is sufficiently large and $\beta_{\max }$ is such that

$$
\beta_{\max }<\sqrt[q-1]{\frac{1}{2^{p+q-1}} \frac{C_{p}}{p} \frac{q}{G_{q}}\left(1+r_{k}-\frac{(p-1) \epsilon_{2}^{\frac{p}{p-1}}}{C_{p}}-\frac{\epsilon_{2}^{-p}}{C_{p}}\right)} .
$$


Proof. Let us assume that $u_{k}^{\delta} \in B$, and then applying (3.3) in the first term on the right side to (3.12), we get

$$
\begin{gathered}
\gamma_{k+1}^{\delta}-\gamma_{k}^{\delta} \leq-\frac{\mu}{2}\left\|F\left(u_{k}^{\delta}\right)-v^{\delta}\right\|^{p}+\left(2^{p+q-2} \frac{G_{q}}{q} \beta_{k}^{q}+\beta_{k} \frac{(p-1) \epsilon_{2}^{\frac{p}{p-1}}}{p}\right)\left\|u^{\dagger}-u_{0}\right\|^{p}+\mu\left\|F\left(u_{k}^{\delta}\right)-v^{\delta}\right\|^{p-1} \delta \\
+\frac{\mu}{2} L C_{F}^{2}\left(\frac{p}{C_{p}}\right)^{2 / p}\left\|F\left(u_{k}^{\delta}\right)-v^{\delta}\right\|^{p+\epsilon}+\left(\frac{\beta_{k} \epsilon_{2}^{-p}}{C_{p}}-\beta_{k}\left(1+r_{k}\right)+2^{p+q-2} \beta_{k}^{q} \frac{G_{q}}{q} \frac{p}{C_{p}}\right) \gamma_{k}^{\delta} .
\end{gathered}
$$

From the mean value inequality, (3) of Assumption 3.1, (2.3) and (3.4), we get

$$
\begin{gathered}
\left\|F\left(u_{k}^{\delta}\right)-v^{\delta}\right\|=\left\|F\left(u_{k}^{\delta}\right)-F\left(u^{\dagger}\right)\right\|+\delta \leq \hat{L}\left\|u_{k}^{\delta}-u^{\dagger}\right\|+\delta \leq \hat{L}\left(\frac{p}{C_{p}}\right)^{1 / p} \Delta_{p}^{u_{0}}\left(u^{\dagger}, u_{k}^{\delta}\right)^{1 / p}+\delta \\
\leq \hat{L}\left(\frac{p}{C_{p}}\right)^{1 / p} \rho^{\frac{2}{p}}+\delta \leq\left(\frac{C_{p}}{p}\right)^{\frac{2}{p \epsilon}}\left(L C_{F}^{2}\right)^{\frac{-1}{\epsilon}}+\delta
\end{gathered}
$$

Above with the inequality

$$
(r+s)^{\epsilon} \leq r^{\epsilon}+s^{\epsilon} \text { for } r, s \geq 0, \epsilon \in[0,1],
$$

implies that

$$
\left\|F\left(u_{k}^{\delta}\right)-v^{\delta}\right\|^{\epsilon} \leq\left[\left(\frac{C_{p}}{p}\right)^{\frac{2}{p \epsilon}}\left(L C_{F}^{2}\right)^{\frac{-1}{\epsilon}}+\delta\right]^{\epsilon} \leq\left(\frac{C_{p}}{p}\right)^{\frac{2}{p}}\left(L C_{F}^{2}\right)^{-1}+\delta^{\epsilon} .
$$

This inequality further leads to the estimate

$$
\begin{aligned}
& -\frac{\mu}{2}\left\|F\left(u_{k}^{\delta}\right)-v^{\delta}\right\|^{p}+\frac{\mu}{2} L C_{F}^{2}\left(\frac{p}{C_{p}}\right)^{2 / p}\left\|F\left(u_{k}^{\delta}\right)-v^{\delta}\right\|^{p+\epsilon} \\
& =\left\|F\left(u_{k}^{\delta}\right)-v^{\delta}\right\|^{p}\left[-\frac{\mu}{2}+\frac{\mu}{2} L C_{F}^{2}\left(\frac{p}{C_{p}}\right)^{2 / p}\left\|F\left(u_{k}^{\delta}\right)-v^{\delta}\right\|^{\epsilon}\right] \leq \frac{\mu}{2} L C_{F}^{2}\left(\frac{p}{C_{p}}\right)^{2 / p} \delta^{\epsilon}\left\|F\left(u_{k}^{\delta}\right)-v^{\delta}\right\|^{p} .
\end{aligned}
$$

Employing the estimate

$$
\left(r_{1}+s_{1}\right)^{\lambda} \leq 2^{\lambda-1}\left(r_{1}^{\lambda}+s_{1}^{\lambda}\right) \text { for } r_{1}, s_{1} \geq 0, \lambda \geq 1,
$$

in (3.15) after incorporating (3.14) in it to obtain

$$
\begin{aligned}
-\frac{\mu}{2}\left\|F\left(u_{k}^{\delta}\right)-v^{\delta}\right\|^{p}+\frac{\mu}{2} L C_{F}^{2}\left(\frac{p}{C_{p}}\right)^{2 / p}\left\|F\left(u_{k}^{\delta}\right)-v^{\delta}\right\|^{p+\epsilon} \\
\quad \leq 2^{p-1} \frac{\mu}{2}\left(\delta^{\epsilon}\right)\left(\frac{C_{p}}{p}\right)^{\frac{2(p-\epsilon)}{p \epsilon}}\left(L C_{F}^{2}\right)^{\frac{-(p-\epsilon)}{\epsilon}}+2^{p-1} \frac{\mu}{2} L C_{F}^{2}\left(\frac{p}{C_{p}}\right)^{2 / p} \delta^{p+\epsilon} .
\end{aligned}
$$

Thus, (3.13) and (3.16) imply that

$$
\begin{gathered}
\gamma_{k+1}^{\delta}-\gamma_{k}^{\delta} \leq\left(2^{p+q-2} \frac{G_{q}}{q} \beta_{k}^{q}+\beta_{k} \frac{(p-1) \epsilon_{2}^{\frac{p}{p-1}}}{p}\right)\left\|u^{\dagger}-u_{0}\right\|^{p}+2^{p-1} \frac{\mu}{2} \delta^{\epsilon}\left(\frac{C_{p}}{p}\right)^{\frac{2(p-\epsilon)}{p \epsilon}}\left(L C_{F}^{2}\right)^{\frac{-(p-\epsilon)}{\epsilon}}+ \\
\left(\frac{\beta_{k} \epsilon_{2}^{-p}}{C_{p}}-\beta_{k}\left(1+r_{k}\right)+2^{p+q-2} \beta_{k}^{q} \frac{G_{q}}{q} \frac{p}{C_{p}}\right) \gamma_{k}^{\delta}+\mu\left\|F\left(u_{k}^{\delta}\right)-v^{\delta}\right\|^{p-1} \delta+2^{p-1} \frac{\mu}{2} L C_{F}^{2}\left(\frac{p}{C_{p}}\right)^{2 / p} \delta^{p+\epsilon} .
\end{gathered}
$$


Because of the assumption $u_{0} \in B$ and (2.3), estimate (3.17) can be rewritten as

$$
\begin{array}{r}
\gamma_{k+1}^{\delta}-\gamma_{k}^{\delta} \leq\left[\frac{p}{C_{p}}\left(2^{p+q-2} \frac{G_{q}}{q} \beta_{k}^{q}+\beta_{k} \frac{(p-1) \epsilon_{2}^{\frac{p}{p-1}}}{p}\right)+\left(\frac{\beta_{k} \epsilon_{2}^{-p}}{C_{p}}-\beta_{k}\left(1+r_{k}\right)+2^{p+q-2} \beta_{k}^{q} \frac{G_{q}}{q} \frac{p}{C_{p}}\right)\right] \rho^{2} \\
+\mu\left\|F\left(u_{k}^{\delta}\right)-v^{\delta}\right\|^{p-1} \delta+2^{p-1} \frac{\mu}{2} \delta^{\epsilon}\left(\frac{C_{p}}{p}\right)^{\frac{2(p-\epsilon)}{p \epsilon}}\left(L C_{F}^{2}\right)^{\frac{-(p-\epsilon)}{\epsilon}}+2^{p-1} \frac{\mu}{2} L C_{F}^{2}\left(\frac{p}{C_{p}}\right)^{2 / p} \delta^{p+\epsilon} \\
=\left[2^{p+q-1} \beta_{k}^{q} \frac{G_{q}}{q} \frac{p}{C_{p}}+\beta_{k}\left(\frac{(p-1) \epsilon_{2}^{\frac{p}{p-1}}}{C_{p}}+\frac{\epsilon_{2}^{-p}}{C_{p}}-\left(1+r_{k}\right)\right)\right] \rho^{2}+2^{p-1} \frac{\mu}{2} \delta^{\epsilon}\left(\frac{C_{p}}{p}\right)^{\frac{2(p-\epsilon)}{p \epsilon}}\left(L C_{F}^{2}\right)^{\frac{-(p-\epsilon)}{\epsilon}} \\
+\mu\left\|F\left(u_{k}^{\delta}\right)-v^{\delta}\right\|^{p-1} \delta+2^{p-1} \frac{\mu}{2} L C_{F}^{2}\left(\frac{p}{C_{p}}\right)^{2 / p} \delta^{p+\epsilon} .
\end{array}
$$

Now we know that $p>1$ which means either $0<p-1 \leq 1$ or $p-1>1$. If $p-1 \leq 1$, then employ the estimate $(r+s)^{p-1} \leq r^{p-1}+s^{p-1}$ for $r, s \geq 0$, otherwise estimate $(r+s)^{p-1} \leq$ $2^{p-2}\left(r^{p-1}+s^{p-1}\right)$ for $r, s \geq 0$ in (3.14) to obtain (we find a single estimate for both the cases)

$$
\left\|F\left(u_{k}^{\delta}\right)-v^{\delta}\right\|^{p-1} \leq \max \left\{2^{p-2}, 1\right\}\left[\left(\frac{C_{p}}{p}\right)^{\frac{2(p-1)}{p \epsilon}}\left(L C_{F}^{2}\right)^{\frac{-(p-1)}{\epsilon}}+\delta^{p-1}\right] .
$$

Employing this estimate in (3.18) to get

$$
\begin{gathered}
\gamma_{k+1}^{\delta}-\gamma_{k}^{\delta} \leq\left[2^{p+q-1} \beta_{k}^{q} \frac{G_{q}}{q} \frac{p}{C_{p}}+\beta_{k}\left(\frac{(p-1) \epsilon_{2}^{\frac{p}{p-1}}}{C_{p}}+\frac{\epsilon_{2}^{-p}}{C_{p}}-\left(1+r_{k}\right)\right)\right] \rho^{2}+2^{p-1} \frac{\mu}{2} \delta^{\epsilon}\left(\frac{C_{p}}{p}\right)^{\frac{2(p-\epsilon)}{p \epsilon}} \times \\
\left(L C_{F}^{2}\right)^{\frac{-(p-\epsilon)}{\epsilon}}+K_{1} \delta\left(\frac{C_{p}}{p}\right)^{\frac{2(p-1)}{p \epsilon}}\left(L C_{F}^{2}\right)^{\frac{-(p-1)}{\epsilon}}+K_{1} \delta^{p}+2^{p-1} \frac{\mu}{2} L C_{F}^{2}\left(\frac{p}{C_{p}}\right)^{2 / p} \delta^{p+\epsilon},
\end{gathered}
$$

where $K_{1}=\mu \max \left\{2^{p-2}, 1\right\}$. Using the stopping rule discussed in (10) of Assumption 3.1 in (3.19) to obtain

$$
\begin{array}{r}
\gamma_{k+1}^{\delta}-\gamma_{k}^{\delta} \leq\left[2^{p+q-1} \beta_{k}^{q} \frac{G_{q}}{q} \frac{p}{C_{p}}+\beta_{k}\left(\frac{(p-1) \epsilon_{2}^{\frac{p}{p-1}}}{C_{p}}+\frac{\epsilon_{2}^{-p}}{C_{p}}-\left(1+r_{k}\right)\right)\right] \rho^{2}+K_{1} \tau^{-p} \beta_{k}^{p} \\
+K_{2} \tau^{-\epsilon} \beta_{k}^{\epsilon}+K_{3} \tau^{-(p+\epsilon)} \beta_{k}^{p+\epsilon}+K_{4} \tau^{-1} \beta_{k},
\end{array}
$$

where the constants $K_{2}, K_{3}$ and $K_{4}$ are as follows:

$$
\begin{gathered}
K_{2}=2^{p-1} \frac{\mu}{2}\left(\frac{C_{p}}{p}\right)^{\frac{2(p-\epsilon)}{p \epsilon}}\left(K_{5}\right)^{\frac{-(p-\epsilon)}{\epsilon}}, \quad K_{3}=2^{p-1} \frac{\mu}{2} K_{5}\left(\frac{p}{C_{p}}\right)^{2 / p}, K_{5}=L C_{F}^{2}, \\
\text { and } K_{4}=K_{1}\left(\frac{C_{p}}{p}\right)^{\frac{2(p-1)}{p \epsilon}}\left(K_{5}\right)^{\frac{-(p-1)}{\epsilon}} .
\end{gathered}
$$

Observe that under the conditions, $2^{p+q-1} \beta_{k}^{q} \frac{G_{q}}{q} \frac{p}{C_{p}}+\beta_{k}\left(\frac{(p-1) \epsilon_{2}^{\frac{p}{p-1}}}{C_{p}}+\frac{\epsilon_{2}^{-p}}{C_{p}}-\left(1+r_{k}\right)\right)<0, \beta_{\max }$ sufficiently small and $\tau$ sufficiently large, right side of (3.20) can be less than 0 (see Remark 3.5). Since $\tau$ can be taken arbitrary large, for right side of (3.20) to be negative, we must have

$$
\beta_{k}^{q-1}<\frac{1}{2^{p+q-1}} \frac{C_{p}}{p} \frac{q}{G_{q}}\left(1+r_{k}-\frac{(p-1) \epsilon_{2}^{\frac{p}{p-1}}}{C_{p}}-\frac{\epsilon_{2}^{-p}}{C_{p}}\right) .
$$


Therefore, by taking $\beta_{k}$ 's sufficiently smaller than the one's satisfying (3.21), we get

$$
\gamma_{k+1}^{\delta}-\gamma_{k}^{\delta} \leq 0 \Longrightarrow \gamma_{k+1}^{\delta} \leq \gamma_{k}^{\delta} \leq \rho^{2} \Longrightarrow u_{k+1}^{\delta} \in B
$$

Deducing the negativity of the right side of (3.20) is not an easy task because of the involvement of so many constants. We will analyze this condition in a better way by computing some of the constants appearing in it for Banach spaces such as $\mathcal{L}^{p}(\Sigma)$ (Lebesgue integrable functions), $\ell^{p}$ spaces for $p \geq 2$ etc. in Remark 3.5. In the next lemma, we obtain the convergence rates for the iterates of (2.1)-(2.2).

Lemma 3.3. Let the assumptions of Lemma 3.2 hold. Then, we have the following convergence rates for the iterates of (2.1)-(2.2):

$$
\Delta_{p}^{u_{0}}\left(u^{\dagger}, u_{k_{\star}}^{\delta}\right)-\left(1-K_{6}\right) \rho^{2}=O\left(\delta^{\epsilon}\right), \text { as } \delta \rightarrow 0 .
$$

Proof. From (3.20) and (3.21), we get the estimate

$$
\gamma_{k+1}^{\delta} \leq \gamma_{k}^{\delta}+K_{1} \delta^{p}+K_{2} \delta^{\epsilon}+K_{3} \delta^{p+\epsilon}+K_{4} \delta-K_{6} \rho^{2}
$$

with $K_{6}=-2^{p+q-1} \beta_{k}^{q} \frac{G_{q}}{q} \frac{p}{C_{p}}-\beta_{k}\left(\frac{(p-1) \epsilon_{2}^{\frac{p}{p-1}}}{C_{p}}+\frac{\epsilon_{2}^{-p}}{C_{p}}-\left(1+r_{k}\right)\right)>0$. Therefore, for $0<\epsilon \leq 1$, we have

$$
\Delta_{p}^{u_{0}}\left(u^{\dagger}, u_{k_{\star}}^{\delta}\right)-\left(1-K_{6}\right) \rho^{2}=O\left(\delta^{\epsilon}\right), \text { as } \delta \rightarrow 0
$$

Till now, we have proved the results of Theorem 3.1 for noisy data. Now, in the coming lemmas we discuss results for non-noisy data.

Lemma 3.4. Suppose that $\delta=0$ and the assumptions of Lemma 3.2 are satisfied. Then, all the iterates of (2.1)-(2.2) remain in $B$ and converge to the exact solution $u^{\dagger}$. Moreover, iterates satisfy the recurrence relation

$$
\gamma_{k+1} \leq-K_{8} \gamma_{k}^{\frac{2}{1+\epsilon}}+\alpha_{k} \gamma_{k}+K_{11} \beta_{k}
$$

where $K_{8}, K_{11}$ are positive constants and $\left\{\alpha_{k}\right\}$ is a sequence converging to 1 .

Proof. From Lemma 3.2 via (3.19) and (3.21), it is easy to see that all the iterates of (2.1)-(2.2) remain in $B$, sequence $\left\{\gamma_{k}\right\}$ is monotonically decreasing and bounded below by 0 for $\delta=0$, where $\Delta_{p}^{u_{0}}\left(u^{\dagger}, u_{k}\right)=\gamma_{k}$ for each $k$. This means that the limit of the sequence $\left\{\gamma_{k}\right\}$ exists. Let $\lim _{k \rightarrow \infty} \gamma_{k}=a$. We show that the sequence $\left\{\gamma_{k}\right\}$ converges to 0. Putting (3.16) with $\delta=0$ into (3.12) yields

$$
\begin{aligned}
\gamma_{k+1}-\gamma_{k} \leq\left(2^{q-1} \frac{G_{q}}{q} \mu^{q} \hat{L}^{q}-\frac{\mu}{2}\right)\left\|F\left(u_{k}\right)-v\right\|^{p} & +\left(2^{p+q-2} \frac{G_{q}}{q} \beta_{k}^{q}+\beta_{k} \frac{(p-1) \epsilon_{2}^{\frac{p}{p-1}}}{p}\right)\left\|u^{\dagger}-u_{0}\right\|^{p} \\
& +\left(\frac{\beta_{k} \epsilon_{2}^{-p}}{C_{p}}-\left(1+r_{k}\right) \beta_{k}+2^{p+q-2} \beta_{k}^{q} \frac{G_{q}}{q} \frac{p}{C_{p}}\right) \gamma_{k} .
\end{aligned}
$$


We rewrite the above equation as

$$
\begin{aligned}
\gamma_{k+1}-\gamma_{k} \leq-K_{7}\left\|F\left(u_{k}\right)-v\right\|^{p}+\left(2^{p+q-2}\right. & \left.\frac{G_{q}}{q} \beta_{k}^{q}+\beta_{k} \frac{(p-1) \epsilon_{2}^{\frac{p}{p-1}}}{p}\right)\left\|u^{\dagger}-u_{0}\right\|^{p} \\
+ & \left(\frac{\beta_{k} \epsilon_{2}^{-p}}{C_{p}}-\left(1+r_{k}\right) \beta_{k}+2^{p+q-2} \beta_{k}^{q} \frac{G_{q}}{q} \frac{p}{C_{p}}\right) \gamma_{k},
\end{aligned}
$$

where $K_{7}=-2^{q-1} \frac{G_{q}}{q} \mu^{q} \hat{L}^{q}+\frac{\mu}{2}>0$ because of (3.3). Taking limit $k \rightarrow \infty$ and then incorporating $\sum_{k} \beta_{k}<\infty$ and (3.2) in (3.22), we get

$$
a-a \leq-K_{7} \lim _{k \rightarrow \infty}\left\|F\left(u_{k}\right)-v\right\|^{p}+0 \leq-\frac{K_{7}}{\left(C_{F}\right)^{\frac{2 p}{1+\epsilon}}} \lim _{k \rightarrow \infty} \gamma_{k}^{\frac{2}{1+\epsilon}}=-K_{8} \lim _{k \rightarrow \infty} \gamma_{k}^{\frac{2}{1+\epsilon}},
$$

where $K_{8}=\frac{K_{7}}{\left(C_{F}\right)^{\frac{2 p}{1+\epsilon}}}$ is a positive constant. Now, using the continuity of the function $x \rightarrow x^{a}$ for any $a>1,(3.23)$ implies that

$$
0 \leq-K_{8} a^{\frac{2}{1+\epsilon}} \Longrightarrow a^{\frac{2}{1+\epsilon}} \leq 0 .
$$

But as $\gamma_{k} \geq 0$, we must have $a \geq 0$ and thus above implies that $a=0$. Hence, by (4) in Theorem $2.2, u_{k} \rightarrow u^{\dagger}$, i.e. iterates of (2.1)-(2.2) converges to the exact solution for non-noisy data. Next, we find the recursion formula satisfied by the sequence $\left\{\gamma_{k}\right\}$. Using (3.2) and $u_{0} \in B$ in (3.22) to reach at

$$
\begin{gathered}
\gamma_{k+1} \leq-K_{8} \gamma_{k}^{\frac{2}{1+\epsilon}}+\left(2^{p+q-2} \frac{G_{q}}{q} \beta_{k}^{q}+\beta_{k} \frac{(p-1) \epsilon_{2}^{\frac{p}{p-1}}}{p}\right) \frac{p}{C_{p}} \rho^{2}+ \\
\left(1+\frac{\beta_{k} \epsilon_{2}^{-p}}{C_{p}}-\left(1+r_{k}\right) \beta_{k}+2^{p+q-2} \beta_{k}^{q} \frac{G_{q}}{q} \frac{p}{C_{p}}\right) \gamma_{k} \\
=-K_{8} \gamma_{k}^{\frac{2}{1+\epsilon}}+\alpha_{k} \gamma_{k}+K_{9} \beta_{k}^{q}+K_{10} \beta_{k} \leq-K_{8} \gamma_{k}^{\frac{2}{1+\epsilon}}+\alpha_{k} \gamma_{k}+K_{11} \beta_{k}
\end{gathered}
$$

where constants $K_{9}, K_{10}$ and $\alpha_{k}$ are as follows:

$K_{9}=2^{p+q-2} \frac{G_{q}}{q} \frac{p}{C_{p}} \rho^{2}, \quad K_{10}=\frac{(p-1) \epsilon_{2}^{\frac{p}{p-1}}}{C_{p}} \rho^{2}, \quad \alpha_{k}=1+\frac{\beta_{k} \epsilon_{2}^{-p}}{C_{p}}-\beta_{k}\left(1+r_{k}\right)+2^{p+q-2} \beta_{k}^{q} \frac{G_{q}}{q} \frac{p}{C_{p}}$, and the last term in (3.24) is written because $\beta_{k}<1$ and $q>1$, where $K_{11}=K_{9}+K_{10}$. We can easily see that $\alpha_{k} \rightarrow 1$. So, (3.24) is the required recurrence relation.

In the next lemma, we obtain the convergence rates for noise-free iterates in terms of radius $\rho$ of $B$.

Lemma 3.5. Let the assumptions of Lemma 3.4 hold and there exists a constant $C \geq 0$ such that $\beta_{k} \leq C \gamma_{k}$ for each $k$. Then, for $\epsilon \in(0,1)$ we have the following convergence rate

$$
\Delta_{p}^{u_{0}}\left(u^{\dagger}, u_{k}\right) \leq\left(\left(g_{k} \rho^{2}\right)^{-\frac{1-\epsilon}{1+\epsilon}}+h_{k}\right)^{-\frac{1+\epsilon}{1-\epsilon}}, \quad k=1,2, \ldots
$$

For $\epsilon=1$, we have

$$
\Delta_{p}^{u_{0}}\left(u^{\dagger}, u_{k}\right) \leq \prod_{i=0}^{k-1}\left(-K_{8}+\alpha_{i}+K_{11} C\right) \rho^{2}, \quad k=1,2, \ldots
$$


(see proof for the meaning of constants $g_{k}, h_{k}$ for $k \geq 1$ ).

Proof. With the given condition $\beta_{k} \leq C \gamma_{k},(3.24)$ can be written as

$$
\begin{array}{r}
\gamma_{k+1} \leq-K_{8} \gamma_{k}^{\frac{2}{1+\epsilon}}+\alpha_{k} \gamma_{k}+K_{11} \beta_{k} \leq-K_{8} \gamma_{k}^{\frac{2}{1+\epsilon}}+d_{k} \gamma_{k} \\
=d_{k} \gamma_{k}\left(1-e_{k} \gamma_{k}^{\frac{1-\epsilon}{1+\epsilon}}\right),
\end{array}
$$

where $d_{k}=\alpha_{k}+C K_{11}$ and $e_{k}=\frac{K_{8}}{d_{k}}$ for every $k$. Let $t=\frac{1-\epsilon}{1+\epsilon}$. Then, (3.25) yields

$$
\left(\gamma_{k+1}\right)^{-t} \geq\left(d_{k} \gamma_{k}\right)^{-t}\left(1-e_{k} \gamma_{k}^{t}\right)^{-t}
$$

Applying the estimate $(1-y)^{-t} \geq 1+t y, \forall y \in(0,1)$ into (3.26) for $k \geq 0$, we get

$$
\left(\gamma_{k+1}\right)^{-t} \geq\left(d_{k} \gamma_{k}\right)^{-t}+f_{k}
$$

where $f_{k}=t e_{k} d_{k}^{-t}$. Thus, we have

$$
\Delta_{p}^{u_{0}}\left(u^{\dagger}, u_{k}\right) \leq\left(\left(g_{k} \rho^{2}\right)^{-\frac{1-\epsilon}{1+\epsilon}}+h_{k}\right)^{-\frac{1+\epsilon}{1-\epsilon}}, \quad k=1,2, \ldots
$$

where

$$
g_{k}=\prod_{i=0}^{k-1} d_{i}, \text { for } k \geq 1
$$

and

$$
h_{k}=\sum_{j=1}^{k-1}\left(d_{j} d_{j+1} \ldots d_{k-1}\right)^{-\frac{1+\epsilon}{1-\epsilon}} f_{j-1}+f_{k-1}, k \geq 2, h_{1}=f_{0} .
$$

For $\epsilon=1,(3.25)$ with $\beta_{k} \leq C \gamma_{k}$ implies that

$$
\gamma_{k} \leq \prod_{i=0}^{k-1}\left(-K_{8}+\alpha_{i}+K_{11} C\right) \rho^{2}, \quad k=1,2, \ldots
$$

So, we get the convergence rates via in terms of radius $\rho$ of $B$.

Remark 3.4. The condition $\beta_{k} \leq C \gamma_{k}$ assumed in Lemma 3.5 is an abstract smoothness condition for obtaining the convergence rates and is similar to other smoothness concepts (e.g. source conditions, variational inequalities) already available in the literature $[4,16,26,27,33]$ in the sense that all these incorporate some a-priori knowledge of the exact solution.

For the noise free iterates of (2.1)-(2.2), we also obtain convergence rates in terms of $\beta_{k}$ 's where $\beta_{k}$ satisfy $(2.2)$ for each $k$.

Lemma 3.6. In addition to the rates obtained in Lemma 3.5, we also obtain the rates

$$
\Delta_{p}^{u_{0}}\left(u^{\dagger}, u_{k}\right)=O\left(\beta_{k}^{q-1}\right) \quad \text { as } k \rightarrow \infty,
$$

provided the assumptions of Lemma 3.4 hold and

$$
K_{12}+\eta \beta_{k}^{-1}\left[\alpha_{k}-\left(\frac{\beta_{k+1}}{\beta_{k}}\right)^{q-1}\right] \leq 0
$$


where $\eta, K_{12}$ are positive constants and $\left\{\alpha_{k}\right\}$ is a sequence converging to 1 .

Proof. The inequality (3.24) leads to the estimate

$$
\gamma_{k+1} \leq-K_{8} \gamma_{k}^{\frac{2}{1+\epsilon}}+\alpha_{k} \gamma_{k}+K_{12} \beta_{k}^{q}
$$

where $K_{12}$ is such that $K_{12} \beta_{k}^{q}>K_{11} \beta_{k}$ for every $k$ (such a condition is possible as $\beta_{\max }<\infty$ ). Now, let us define $\eta_{k}=\frac{\gamma_{k}}{\beta_{k}^{q-1}}$. Then from (3.27), we have

$$
\eta_{k+1} \leq\left(\frac{\beta_{k}}{\beta_{k+1}}\right)^{q-1}\left[-K_{8} \eta_{k}^{1+t} \beta_{k}^{(q-1) t}+\alpha_{k} \eta_{k}+K_{12} \beta_{k}\right] \leq\left(\frac{\beta_{k}}{\beta_{k+1}}\right)^{q-1}\left[\alpha_{k} \eta_{k}+K_{12} \beta_{k}\right],
$$

where $t=\frac{1-\epsilon}{1+\epsilon}$. For the uniform boundedness of $\left\{\eta_{k}\right\}$ by some $\eta$, sufficient condition is

$$
\alpha_{k} \eta+K_{12} \beta_{k} \leq \eta\left(\frac{\beta_{k+1}}{\beta_{k}}\right)^{q-1} \Longrightarrow K_{12}+\eta \beta_{k}^{-1}\left[\alpha_{k}-\left(\frac{\beta_{k+1}}{\beta_{k}}\right)^{q-1}\right] \leq 0 .
$$

Thus, we have $\Delta_{p}^{u_{0}}\left(u^{\dagger}, u_{k}\right)=\gamma_{k}=O\left(\beta_{k}^{q-1}\right)$ as $k \rightarrow \infty$.

On combining Lemmata 3.1-3.6, one can see that proof of the Theorem 3.1 is complete. Observe that, for $0<\epsilon<1$, in the case of non-noisy data we have obtained the sub-linear convergence rates in Lemma 3.5 and as $\epsilon \rightarrow 1$, speed of the convergence increases because it switches to the linear convergence. Further, in Lemma 3.6 we have obtained the rates in terms of $\beta_{i}$ 's and rates are sub-linear or super-linear accordingly as $1<q<2$ or $q>2$ respectively.

For proving Theorem 3.1 (especially Lemma 3.2), we require the condition $2^{p+q-1} \beta_{k}^{q} \frac{G_{q}}{q} \frac{p}{C_{p}}+$ $\beta_{k}\left(\frac{(p-1) \epsilon_{2}^{\frac{p}{p-1}}}{C_{p}}+\frac{\epsilon_{2}^{-p}}{C_{p}}-\left(1+r_{k}\right)\right)<0$. We discuss about this condition in the following remark.

Remark 3.5. For (3.21) to be satisfied, we must have the following:

(i) $2^{p+q-1} \beta_{k}^{q-1} \frac{G_{q}}{q} \frac{p}{C_{p}}<1+r_{k}-\frac{(p-1) \epsilon_{2}^{\frac{p}{p-1}}}{C_{p}}-\frac{\epsilon_{2}^{-p}}{C_{p}}$, for each $k$.

(ii) $\frac{(p-1) \epsilon_{2}^{\frac{p}{p-1}}}{C_{p}}+\frac{\epsilon_{2}^{-p}}{C_{p}}<1+r_{k}$, for each $k$.

Observe that $(i)$ can be easily handled by taking $\beta_{\max }$ sufficiently small provided (ii) holds. For (ii), first, we find the values of $C_{p}$ for different $p$. Using (2.7), we get the following table (values of $K_{p}$ can be obtained by writing a simple program in MATLAB, C++ etc.):

\begin{tabular}{|l|l|}
\hline Value of $p$ & Value of $K_{p}$ (approx.) \\
\hline 1.5 & 3.8132 \\
\hline 2 & 5.2086 \\
\hline 3 & 7.3326 \\
\hline 4 & 8.9576 \\
\hline 5 & 10.2274 \\
\hline$\ldots$ & $\ldots$ \\
\hline 10 & 13.4980 \\
\hline
\end{tabular}


TABLE 1. Relationship between $p$ and $K_{p}$

From Remark 2.4, we know that $C_{p}=\frac{Y K_{p}}{2^{p}}$, where the constant $Y$ is same as appearing in the Definition 2.4. Now, if $U=\mathcal{L}^{p}(\Sigma)$, where $p \geq 2$ and $\Sigma \subset \mathbb{R}^{n}$ is an open domain, then from Example 2.2 we know that $\delta_{U}(\epsilon)=\epsilon^{p}$ for any $\epsilon \in(0,2]$. In other words, $U$ is $p$ convex for any $p \geq 2$ with $Y=1$. Therefore,

$$
C_{p}=\frac{K_{p}}{2^{p}}, \quad \text { for } U=\mathcal{L}^{p}(\Sigma)
$$

So, (ii) holds provided

$$
(p-1) \epsilon_{2}^{\frac{p}{p-1}}+\epsilon_{2}^{-p}<\frac{\left(1+r_{k}\right) K_{p}}{2^{p}} .
$$

For instance, take $p=2$ and $\epsilon_{2}=1$. Then the last inequality becomes

$$
\epsilon_{2}^{2}+\epsilon_{2}^{-2}=2<\frac{(1+1)(5.2086)}{4},
$$

which is true since $r_{k}=1$ for each $k$ (see (6) of Assumption 3.1). This means our assumption of Lemma 3.2 related to $\beta_{k}$ is satisfied. The rationale behind introducing $\epsilon_{2}$ in (3.10) is to make the estimate (ii) more flexible so that it holds for a range of values of $r_{k}$ or $\epsilon_{2}$. In other words one can see that (ii) also holds for $p=2$ and $\epsilon_{2}=0.9$. Further, from [38, Equation 1.2] we can see that the Banach spaces $\ell^{p}, W_{m}^{p}$ (Sobolev space) are $\max \{2, p\}$ convex and one can verify the condition (ii) provided exact bound for $Y$ is known as in the case of $\mathcal{L}^{p}$ spaces.

Next result is for the crucial case when $\epsilon=0$ in (3.2). We need to do this case separately as we can not take $\epsilon=0$ in the proof of Theorem 3.1 (see (3.16), which contains a term having $\epsilon$ in the denominator). For the case $\epsilon=0$, we need to have a different bound on $\mu$ than what is assumed in Theorem 3.1 ((8) of Assumption 3.1).

Theorem 3.2. Let $F$ be a non-linear operator between the Banach spaces $U, V$ and the operator equation $F(u)=v, v \in V$, has a solution $u^{\dagger}$. Let the conditions (1)-(7), (10) of Assumption 3.1 hold with $\epsilon=0$ in (5), and $\mu$ satisfies

$$
\mu^{q-1}<\frac{q}{2^{q-1} G_{q} \hat{L}^{q}}\left[1-\frac{1}{2} L C_{F}^{2}\left(\frac{p}{C_{p}}\right)^{2 / p}\right] .
$$

Suppose that $v^{\delta} \in V$ be such that $\left\|v^{\delta}-v\right\| \leq \delta$. Then all the iterates $u_{k+1}^{\delta}$ of iteratively regularized Landweber iteration method (2.1)-(2.2) remain in $B$ for all $k \leq k_{\star}(\delta)-1$ provided (3.21) holds. Moreover, iterates satisfy the following recurrence relation

$$
\gamma_{k+1}^{\delta} \leq \gamma_{k}^{\delta}+M_{1} \delta+M_{2} \delta^{p}-K_{6} \rho^{2}
$$

and we obtain the following convergence rate

$$
\Delta_{p}^{u_{0}}\left(u^{\dagger}, u_{k_{\star}}\right)-\left(1-K_{6}\right) \rho^{2}=O(\delta), \text { as } \delta \rightarrow 0,
$$

for some constants $M_{i}, 1 \leq i \leq 2$, where $\gamma_{k}=\Delta_{p}^{u_{0}}\left(u^{\dagger}, u_{k}^{\delta}\right)$ and constant $K_{6}$ has same meaning as in Theorem 3.1. 
Proof. It can be observed that Lemma 3.1 is valid with our assumptions. So, put $\epsilon=0$ in (3.12) and employ the condition (3.28) in it to obtain

$$
\begin{aligned}
\gamma_{k+1}^{\delta}-\gamma_{k}^{\delta} \leq \mu \delta\left\|F\left(u_{k}^{\delta}\right)-v^{\delta}\right\|^{p-1} & +\left(2^{p+q-2} \frac{G_{q}}{q} \beta_{k}^{q}+\beta_{k} \frac{(p-1) \epsilon_{2}^{\frac{p}{p-1}}}{p}\right)\left\|u^{\dagger}-u_{0}\right\|^{p} \\
& +\left(\frac{\beta_{k} \epsilon_{2}^{-p}}{C_{p}}-\left(1+r_{k}\right) \beta_{k}+2^{p+q-2} \beta_{k}^{q} \frac{G_{q}}{q} \frac{p}{C_{p}}\right) \gamma_{k}^{\delta} .
\end{aligned}
$$

Let us assume that $u_{k}^{\delta} \in B$, then (3.29) with (2.3) leads to

$$
\gamma_{k+1}^{\delta}-\gamma_{k}^{\delta} \leq \mu \delta\left\|F\left(u_{k}^{\delta}\right)-v^{\delta}\right\|^{p-1}+\left[2^{p+q-1} \beta_{k}^{q} \frac{G_{q}}{q} \frac{p}{C_{p}}+\beta_{k}\left(\frac{(p-1) \epsilon_{2}^{\frac{p}{p-1}}}{C_{p}}+\frac{\epsilon_{2}^{-p}}{C_{p}}-\left(1+r_{k}\right)\right)\right] \rho^{2} .
$$

From (3.14) we know that

$$
\left\|F\left(u_{k}^{\delta}\right)-v^{\delta}\right\| \leq \hat{L}\left(\frac{p}{C_{p}}\right)^{1 / p} \rho^{\frac{2}{p}}+\delta .
$$

As $p-1$ is either $\leq 1$ or $>1$, above with the estimate

$$
\left(r_{1}+s_{1}\right)^{\lambda} \leq 2^{\lambda-1}\left(r_{1}^{\lambda}+s_{1}^{\lambda}\right) \text { for } r_{1}, s_{1} \geq 0 \text { and } \lambda \geq 1,
$$

or

$$
\left(r_{1}+s_{1}\right)^{\lambda} \leq r_{1}^{\lambda}+s_{1}^{\lambda} \text { for } r_{1}, s_{1} \geq 0 \text { and } 0 \leq \lambda \leq 1,
$$

accordingly as $p-1 \leq 1$ or $p-1 \geq 1$ with (3.30) imply that

$$
\begin{aligned}
\gamma_{k+1}^{\delta}-\gamma_{k}^{\delta} \leq\left[2^{p+q-1} \beta_{k}^{q} \frac{G_{q}}{q} \frac{p}{C_{p}}+\beta_{k}\left(\frac{(p-1) \epsilon_{2}^{\frac{p}{p-1}}}{C_{p}}\right.\right. & \left.\left.+\frac{\epsilon_{2}^{-p}}{C_{p}}-\left(1+r_{k}\right)\right)\right] \rho^{2}+K_{1} \mu \delta^{p} \\
& +K_{1} \mu \delta \hat{L}^{p-1}\left(\frac{p}{C_{p}}\right)^{\frac{p-1}{p}} \rho^{\frac{2(p-1)}{p}},
\end{aligned}
$$

where $K_{1}=\max \left\{1,2^{p-2}\right\}$. Use the stopping rule discussed in (10) of Assumption 3.1 in above to obtain

$$
\begin{gathered}
\gamma_{k+1}^{\delta}-\gamma_{k}^{\delta} \leq\left[2^{p+q-1} \beta_{k}^{q} \frac{G_{q}}{q} \frac{p}{C_{p}}+\beta_{k}\left(\frac{(p-1) \epsilon_{2}^{\frac{p}{p-1}}}{C_{p}}+\frac{\epsilon_{2}^{-p}}{C_{p}}-\left(1+r_{k}\right)\right)\right] \rho^{2}+M_{1} \delta+M_{2} \delta^{p} \\
\leq\left[2^{p+q-1} \beta_{k}^{q} \frac{G_{q}}{q} \frac{p}{C_{p}}+\beta_{k}\left(\frac{(p-1) \epsilon_{2}^{\frac{p}{p-1}}}{C_{p}}+\frac{\epsilon_{2}^{-p}}{C_{p}}-\left(1+r_{k}\right)\right)\right] \rho^{2}+M_{1} \beta_{k} \tau^{-1}+M_{2} \beta_{k}^{p} \tau^{-p},
\end{gathered}
$$

where $M_{1}=K_{1} \mu \hat{L}^{p-1}\left(\frac{p}{C_{p}}\right)^{\frac{p-1}{p}} \rho^{\frac{2(p-1)}{p}}$ and $M_{2}=K_{1} \mu$. Now if (3.21) holds and $\tau$ is sufficiently large, then we can see that $u_{k+1}^{\delta} \in B$ as in Lemma 3.2. Rest part of the proof follows on the lines of Lemma 3.3 .

Remark 3.6. In Theorem 3.2, we have obtained the convergence rates only for noisy data. However, convergence rates can also be obtained for non-noisy data, in the case when $\epsilon=0$ in (3.2), exactly on the lines of Lemmata 3.4-3.6 as in Theorem 3.1. 
In the following remark, we discuss about the special case when $\left\{\beta_{k}\right\}=\{0\}$. Observe that in this case, (2.1)-(2.2) reduces to Landweber iteration method (non-noisy version) as discussed in Remark 2.3.

Remark 3.7. (i) If $\beta_{k}=0$ for each $k$, then one can reproduce Lemmata 3.1, 3.2, 3.3 to obtain the convergence rates for Landweber iteration method in the case of noisy data which are missing from [24].

(ii) If $\beta_{k}=0$ for each $k$ and $\delta=0$, then observe that the right hand side of (3.19) is trivially satisfied and $\alpha_{k}=0$ (see Lemma 3.4). Also, take $C=0$ in Lemma 3.5 which means $d_{k}=1$, $e_{k}=K_{8}$ and $f_{k}=t K_{8}$ for each $k$. Therefore, one can see that the rates obtained in Lemma 3.5 and the rates obtained in [24] for Landweber iteration method are of the same order. Hence, we can say that the results of [24] can be deduced from our results in a special case.

\section{Electrical Impedance Tomography (EIT)}

In this section, we discuss an example related to Calderón's inverse problem which satisfies the Hölder stability estimate (3.2) under some assumptions on the electrical conductivity. This problem has been also considered in [24] to obtain the convergence and convergence rates of nonlinear Landweber iteration scheme in Banach spaces. Our results on covergence can be applied on the Calderón's inverse problem which is the mathematical bedrock of EIT. It is well known that this Calderón's inverse problem is severely ill-posed [11]. Ulhmann, in [14] has recently studied the EIT and Calderón's problem and further, we refer to [19, 20, 28, 29] for some of the literature in this context. In $[6,11]$, two results on the Lipschitz-type stability estimates have been obtained for the Calderón's inverse conductivity problem provided the a-priori information about the conductivity is known, i.e. it is piecewise constant with a bounded number of unknown values. The difference between these two results is that, in [11] a real valued case is discussed whereas a complex valued case is discussed in [6]. In our work, we consider the real valued case which involves the determination of $v \in H^{1}(\Omega)$ where $v$ satisfies

$$
\left\{\begin{array}{cl}
\operatorname{div}(\gamma \nabla v)=0, & \text { in } \Omega \\
v=g, & \text { on } \partial \Omega .
\end{array}\right.
$$

Here $g \in H^{1 / 2}(\partial \Omega), \Omega \subset \mathbb{R}^{n}, n \geq 2$ is a bounded domain having smooth boundary and $\gamma$ is the positive and bounded function representing the electrical conductivity of $\Omega$. If $\gamma$ is a complex valued function in (4.1), then on subjecting to Dirichlet boundary conditions, (4.1) also appears as the asymptotic limit of an elliptic equation with memory in the study of electrical conduction in biological tissues [6]. Further, Calderón's inverse problem has many applications, for instance, in the fields of nondestructive testing of materials, medical imaging, and therefore, it is an important problem to study. 
The inverse problem associated with EIT is the determination of electrical conductivity $\gamma$ from the information of $\Lambda_{\gamma}$, i.e. the Dirichlet to Neumann map which is defined as

$$
\Lambda_{\gamma}: H^{1 / 2}(\partial \Omega) \rightarrow H^{-1 / 2}(\partial \Omega):\left.g \rightarrow\left(\gamma \frac{\partial v}{\partial \nu}\right)\right|_{\partial \Omega},
$$

where the vector $\nu$ is the outward normal to $\partial \Omega$. The operator $F$ associated with the inverse problem is defined by

$$
F: U \subset \mathcal{L}_{+}^{\infty}(\Omega) \rightarrow \mathbb{L}\left(H^{1 / 2}(\partial \Omega), H^{-1 / 2}(\partial \Omega)\right): F(\gamma)=\Lambda_{\gamma},
$$

where $\mathbb{L}\left(H^{1 / 2}(\partial \Omega), H^{-1 / 2}(\partial \Omega)\right)$ is the space of all bounded linear operators from $H^{1 / 2}(\partial \Omega)$ to $H^{-1 / 2}(\partial \Omega)$. Further, $F^{\prime}$, the Fréchet derivative of $F$ at $\gamma=\gamma^{\prime}$ is given by

$$
F^{\prime}\left(\gamma^{\prime}\right): U \subset \mathcal{L}^{\infty}(\Omega) \rightarrow \mathbb{L}\left(H^{1 / 2}(\partial \Omega), H^{-1 / 2}(\partial \Omega)\right): \delta \gamma \rightarrow F^{\prime}\left(\gamma^{\prime}\right)(\delta \gamma),
$$

where $F^{\prime}\left(\gamma^{\prime}\right)(\delta \gamma)$ is defined by the sesquilinear form

$$
\left\langle F^{\prime}\left(\gamma^{\prime}\right)(\delta \gamma) g_{1}, g_{2}\right\rangle=\int_{\Omega} \delta \gamma \nabla v_{1} \cdot \nabla v_{2} d x, \quad g_{1}, g_{2} \in H^{1 / 2}(\partial \Omega),
$$

where $v_{1}$ and $v_{2}$ are the weak solutions of

$$
\begin{cases}\operatorname{div}\left(\gamma^{\prime} \nabla v_{1}\right)=0=\operatorname{div}\left(\gamma^{\prime} \nabla v_{2}\right), & \text { in } \Omega \\ v_{1}=g_{1}, \quad v_{2}=g_{2} & \text { on } \partial \Omega .\end{cases}
$$

Under the assumption that $\gamma \in L^{\infty}(\Omega)$, for the case $n=2$, uniqueness of the solution to the inverse problem (4.2) is discussed in [20] and for $n \geq 3$, it is considered in [22] provided $\gamma$ is in the Sobolev space $W^{3 / 2, \infty}(\Omega)$.

Remaining discussion of this section is mainly based on the results of [11]. So, we refer this article whenever needed instead of recalling all the results. Next theorem presents the Lipschitz estimate established in [11].

Theorem 4.1. Let $\gamma_{1}, \gamma_{2}$ be two real piecewise constant functions such that

$$
\gamma_{i}(x)=\sum_{j=1}^{N} \gamma_{j}^{i}(x) \chi_{D_{j}}(x), x \in \Omega, \lambda \leq \gamma_{i}(x) \leq \lambda, i=1,2,
$$

where $\lambda \in(0,1], \gamma_{j}^{i}$ is an unknown real number for each $i, j, D_{j}$ 's are known open sets, $\chi_{D_{j}}$ is characteristics function of the set $D_{j}$ and $N \in \mathbb{N}$. Then under some assumptions on $\Omega, D_{j}$ 's (see section 2.2 in [11]), we have

$$
\left\|\gamma_{1}-\gamma_{2}\right\|_{L^{\infty}(\Omega)} \leq C\left\|\Lambda_{\gamma_{1}}-\Lambda_{\gamma_{2}}\right\|_{L\left(H^{1 / 2}(\partial \Omega), H^{-1 / 2}(\partial \Omega)\right)}
$$

where $C$ is a constant.

Now, we verify that the assumptions of Theorem 3.1 are satisfied. Before that, observe that the Banach space $L^{\infty}(\Omega)$ is not a uniformly convex space, so defining the space $U$ in accodance with Theorem 4.1 as

$$
U=\operatorname{span}\left\{\chi_{D_{1}}, \chi_{D_{2}}, \ldots, \chi_{D_{N}}\right\}
$$


fitted with $L^{p}$ norm where $p>1, D_{i}$ 's, $\chi_{D_{i}}^{\prime} s$ are same as in Theorem 4.1. Then, with the help of basis $\left\{\chi_{D_{1}}, \chi_{D_{2}}, \ldots, \chi_{D_{N}}\right\}$, one can show the Lipschitz continuity of $F^{\prime}$ and its boundedness [24, subsection 5.3], i.e. (2), (3) in Assumption 3.1.

Further, assume that $v=F\left(\gamma^{\dagger}\right)$ where $\gamma^{\dagger} \in U$. Then (5) of Assumption 3.1 holds (see Theorem 4.1). As the notion of weak and strong topology is equivalent for the finite dimensional spaces, $F$ defined in (4.2) is weakly sequentially closed which means (4) of Assumption 3.1 holds. And let $u_{0},\left\{\beta_{k}\right\}, \mu, \rho^{2}$ and $k_{*}$ are chosen in accordance with Theorem 3.1, then iteratively regularized Landweber iteration method converges in accordance with Theorem 3.1 and we also get the said convergence rates.

Remark 4.1. It is of possible impression that the inverse problem (4.2) becomes well posed by considering the unknown conductivities in a finite dimensional space in Theorem 4.1. However, a counter example to discomfort such an impression is discussed in [11]. We recall that example in our work for the sake of completeness. Let $F: \mathbb{R} \rightarrow \mathbb{R}^{3}$ be such that

$$
F(t)=((2+\cos 3 \pi \alpha t) \cos 2 \pi t,(2+\cos 3 \pi \alpha t) \sin 2 \pi t, \sin 2 \pi \alpha t), t \in \mathbb{R},
$$

where $\alpha$ is a parameter. It can be shown that $F$ is smoothly locally invertible. For $\alpha$ rational, $F$ is periodic whereas for $\alpha$ irrational, it is globally one-to-one but $F^{-1}$ is discontinuous at every point. Further, if $\alpha$ is irrational and $F$ is restricted to interval $[-I, I]$ for some $I>0$, then $F^{-1}$ is globally Lipschitz, but Lipschitz constant may blow up as $\alpha$ tends to any rational number.

\section{Conclusion}

We have implemented the iteratively regularized Landweber iteration scheme for non-linear inverse problems in Banach spaces to obtain the convergence rates. Under the condition that non-linear operator satisfies Hölder stability estimate, we proved the convergence for non-noisy iterates and obtained the sublinear convergence rates under some additional assumptions. To the best of our knowledge, this paper is the first advancement to find the explicit reconstructions for Iteratively regularized landweber iteration method by employing the Hölder stability estimates after the reconstructions in [24]. An important thing to note is that while obtaining the convergence rates for iteratively regularized Landweber iteration method (2.1)-(2.2), complementary, we also get the convergence rates for Landweber scheme (see Remark 3.7) in the presence of noisy data which are missing from the literature.

An important future work in this direction is to come up with a situation where the assumptions of Theorem 3.1 are satisfied in an infinite dimensional Banach space, for instance, one can think of fitting $L^{\infty}(\Omega)$ in some infinite dimensional space in Example 4.1. Further, one can also think about the application of results of Theorem 3.1 in option pricing theory (OPT), see e.g. [37]. The inverse problem associated with OPT is addressed in [32]. 


\section{REFERENCES}

[1] A. B. Bakushinsii, The problems of the convergence of the iteratively regularized Gauss-Newton method, Comput. Math. Math. Phys., 32, 1353 - 1359, 1992.

[2] A. B. Bakushinsii and M. Y. Kokurin, Iterative methods for approximate solution of inverse problems, Springer, 2004.

[3] B. Kaltenbacher, Convergence rates for the iteratively regularized Landweber iteration in Banach space, IFIP Conference on System Modeling and Optimization, vol 391, Springer, 2013.

[4] B. Kaltenbacher, A. Neubauer and O. Scherzer, Iterative regularization methods for nonlinear Illposed problems, De Gruyter, 2008.

[5] C. E. Chidume and H. Zegeye, Global iterative schemes for accretive operators, J. Math. Anal. Appl., $257(2), 364-377,2001$.

[6] E. Beretta and E. Francini, Lipschitz stability for the electrical impedance tomography problem: the complex case, Commun. Part. Differ. Eq., 36(10), 1723 - 1749, 2011.

[7] E. Somersalo, D. Isaacson and M. Cheney, A linearized inverse boundary value problem for Maxwell's equations, J. Comput. Appl. Math., 42, 123 - 136, 1992.

[8] E. Zeidler, Nonlinear functional analysis and its applications II/A. Linear monotone operators, Springer-Verlag, 1990.

[9] E. Zeidler, Nonlinear functional analysis and its applications II/B. Nonlinear monotone operators, Springer-Verlag, 1990.

[10] F. Schöpfer, A. K. Louis and T. Schuster, Nonlinear iterative methods for linear ill-posed problems in Banach spaces, Inverse Probl., 22(1), 311 - 329, 2006.

[11] G. Alessandrini and S. Vessella, Lipschitz stability for the inverse conductivity problem, Adv. in Appl. Math., 35(2), 207 - 241, 2005.

[12] G. Bao and K. Yun, On the stability of an inverse problem for the wave equation, Inverse Probl., 25(4), 045003, pp. 7, 2009.

[13] G. Bao, Y. Chen and F. Ma, Regularity and stability for the scattering map of a linearized inverse medium problem, J. Math. Anal. Appl., 247, 255 - 271, 2000.

[14] G. Uhlmann, Electrical impedance tomography and Calderón's problem, Inverse Probl., 25(12), 123011, 2009.

[15] H. Bellout, A. Friedman and V. Isakov, Stability for an inverse problem in potential theory, Trans. Amer. Math. Soc., 332(1), 271 - 296, 1992.

[16] H. W. Engl, M. Hanke and A. Neubauer, Regularization of inverse problems, Kluwer Academic publishers, 1996.

[17] I. Cioranescu, Geometry of Banach spaces, duality mappings and nonlinear problems, Dordrecht: Kluwer, 1990.

[18] J. Cheng and M. Yamamoto, One new strategy for a priori choice of regularization parameters in Tikhonov's regularization, Inverse Probl., 16(4), L31-L38, 2000.

[19] J. Sylvester and G. Uhlmann, A global uniqueness theorem for an inverse boundary value problem, Ann. of Math., 125(1), 153 - 169, 1987. 
[20] K. Astala and L. Päivärinta, Calderón's inverse conductivity problem in the plane, Ann. of Math., $163,265-299,2006$.

[21] L. H. Landweber, An iteration formula for Fredholm integral equations of first kind, Amer. J. Math., 73(3), $615-624,1951$.

[22] L. Päivärinta, A. Panchenko and G. Uhlmann, Complex geometrical optics solutions for Lipschitz conductivities, Rev. Mat. Iberoam., 19(1), 57 - 72, 2003.

[23] M. Hanke, A. Neubauer and O. Scherzer, A convergence analysis of the Landweber iteration for nonlinear ill-posed problems, Numer. Math., 72(1), 21 - 37, 1995.

[24] M. V. de Hoop, L. Qiu and O. Scherzer, Local analysis of inverse problems: Hölder stability and iterative reconstruction, Inverse Probl., 28, 045001, pp. 16, 2012.

[25] O. Scherzer, A modified Landweber iteration for solving parameter estimation problems, Appl. Math. Optim., 38(1), $45-68,1998$.

[26] O. Scherzer, M. Grasmair, H. Grossauer, M. Haltmeier and F. Lenzen, Variational methods in imaging, Springer, 2009.

[27] R. Andreev, P. Elbau, M. V. de Hoop, L. Qiu, and O Scherzer, Generalized convergence rates results for linear inverse problems in Hilbert spaces, Numer. Funct. Anal. Optim., 36, 549 - 566, 2015.

[28] R. Kohn and M. Vogelius, Determining conductivity by boundary measurements, Commun. Pure Appl. Math., 37(3), 289 - 298, 1984.

[29] R. Kohn and M. Vogelius, Determining conductivity by boundary measurements: II. Interior results, Commun. Pure Appl. Math., 38(5), 643 - 667, 1985.

[30] S. I. Kabanikhin, Inverse and ill-Posed problems: theory and applications, De Gruyter, 2011.

[31] S. Osher, M. Burger, D. Goldfarb, J. Xu and W. Yin, An iterative regularization method for total variation-based image restoration, Multiscale Model. Simul., 4(2), 460 - 489, 2005.

[32] T. Hein and B. Hofmann, On the nature of ill-posedness of an inverse problem arising in option pricing, Inverse Probl., 19(6), 1319 - 1338, 2003.

[33] T. Schuster, B. Kaltenbacher, B. Hofmann and K. S. Kazimierski, Regularization methods in Banach spaces, De Gruyter Publishers, 2012.

[34] V. Isakov, Stability estimates for obstacles in inverse scattering, J. Comput. Appl. Math., 42(1), $79-88,1992$.

[35] V. Isakov, New stability results for soft obstacles in inverse scattering, Inverse Probl., 9(5), 535 - 543, 1993.

[36] Y. I. Alber, A. G. Kartsatos and E. Litsyn, Iterative solution of unstable variational inequalities on approximately given sets, Abstr. Appl. Anal., 1(1), 45 - 64, 1996.

[37] Y. Kwok, Mathematical models of Financial derivatives, Springer-Verlag, 2008.

[38] Z.-B. Xu and G. F. Roach, Characteristic inequalities of uniformly convex and uniformly smooth Banach spaces, J. Math. Anal. Appl., 157(1), 189 - 210, 1991. 